\title{
Highly efficient siRNA transfection in macrophages using apoptotic body-mimic Ca-PS lipopolyplex
}

This article was published in the following Dove Press journal: International Journal of Nanomedicine

Yueyang Lai',*

Xuebo $\mathrm{Xu}^{\mathrm{l}, *}$

Zhenyu Zhu'

Zichun Hua ${ }^{1,2}$

'The State Key Laboratory of Pharmaceutical Biotechnology, School of Life Sciences, Nanjing University, Nanjing, 2 10023 , China; ${ }^{2}$ Changzhou High-Tech Research Institute of Nanjing University and Jiangsu Target Pharma Laboratories Inc., Changzhou, China

*These authors contributed equally to this work
Correspondence: Zichun Hua The State Key Laboratory of Pharmaceutical Biotechnology, School of Life Sciences, Nanjing University, \#163 Xianlian Boulevard, Nanjing 210023, China

Tel +862589683295

Email zchua@nju.edu.cn
Background: The discovery and development of RNA interference has made a tremendous contribution to the biochemical and biomedical field. However, liposomal transfection protocols to deliver siRNAs to certain types of cells, eg, immune cells, are not viable due to exceedingly low transfection efficiency. While viral delivery and electroporation are two widely adopted approaches to transfect immune cells, they are associated with certain drawbacks such as complexity of preparation, biosafety issues, and high cytotoxicity. We believe amendments can be made to liposomal formulas and protocols to achieve a highly efficient knockdown of genes by liposome-loaded siRNAs.

Aim: The aim of this study was to use the apoptotic-mimic Ca-PS lipopolyplex to achieve highly efficient siRNA knockdown of genes in the hard-to-transfect macrophages with reduced cytotoxicity and more efficient cellular uptake.

Results: We devised an anionic liposomal formula containing phosphatidylserine to mimic the apoptotic body, the Ca-PS lipopolyplex. Ca-PS lipopolyplex was proven to be capable of delivering and effecting efficient gene knockdown in multiple cell lines at lowered cytotoxicity. Among the two types of macrophages, namely Ana-1 and bone-marrow derived macrophages, Ca-PS lipopolyplex showed an improvement in knockdown efficiency, as high as $157 \%$, over Lipo2000. Further investigations revealed that Ca-PS promotes increased cellular uptake, lysosomal escape and localization of siRNAs to the perinuclear regions in macrophages. Lastly, transfection by Ca-PS lipopolyplex did not induce spontaneous polarization of macrophages.

Conclusion: The apoptotic body-mimic Ca-PS lipopolyplex is a stable, non-cytotoxic liposomal delivery system for siRNAs featuring vastly improved potency for macrophages and lowered cytotoxicity. It is speculated that Ca-PS lipopolyplex can be applied to other immune cells such as T cells and DC cells, but further research efforts are required to explore its promising potentials.

Keywords: siRNA transfection, anionic liposomes, apoptotic body-mimic, macrophages, Ca-PS lipopolyplex

\section{Introduction}

Delivery of short doubled-stranded RNA (dsRNA) molecules into target cells, either in vivo or in vitro, is of pivotal importance in the biotechnology of RNA interference (RNAi). Since the discovery of RNAi, ${ }^{1}$ approaches for siRNA delivery have been continuously developed and optimized to achieve higher efficiency and lower cytotoxicity. To date, a variety of carriers and relevant methods are available for siRNA delivery in target cells, but lipid-based delivery of siRNA remains a highly efficient, biodegradable and affordable approach for general application of siRNA-mediated gene knockdown. ${ }^{2-10}$ However, mainstream cationic transfection agents are associated with several drawbacks such as lipid-induced cytotoxicity and non-applicability in 
hard-to-transfect cells. ${ }^{11-18}$ Among these hard-to-transfect cells, macrophages are notoriously difficult to transfect using liposomes and, therefore, are usually genetically manipulated using viral vectors; but, preparation of these viral vectors could be challenging and presents biosafety threats to researchers. ${ }^{19}$ Macrophages are a type of immune cells specialized in engulfing hazardous biological entities, eg, dead cells, pathogens, cancer cells, bacteria etc. ${ }^{20-23}$ They act as destroyers of "bad" particles as well as antigenpresenters to elicit specific adaptive immune responses. The biological nature of macrophages, which is analogous to that of sentinels and patrollers, renders them almost "immune" to lipid-based genetic modification, which means liposomes are not the appropriate systems for nucleotide delivery to macrophages due to exceptionally low efficiency of genetic overexpression or knockdown. On the other hand, the process of macrophages devouring dead apoptotic cells has been welldelineated. The recognition of "eat-me" signals, presented on the surface of ApoBDs as PS, by macrophages is central to phagocytosis. ${ }^{24}$ PS becomes externalized on apoptotic cells and, therefore, apoBDs (apoBDs are vesicles containing remnants of apoptotic cells and are also derived from apoptotic cells) and serve as anchors for macrophages to initiate the engulfing process, also known as efferocytosis. ${ }^{25}$ Therefore, PS is essential in bringing macrophages to the proximity of apoBDs and triggering the process of engulfment.

The recognition of "eat-me" signals on the surface of apoBDs by macrophages enlightened us to design liposomes containing PS in the form of DOPS so that our liposomes mimic apoBDs. In fact, applications of PS-containing liposomes have been reported previously, eg, paramagnetic imaging of macrophages. ${ }^{26}$ However, genetic manipulation, employing PS-containing liposomes, is yet to be explored. We designed and produced an anionic liposomal delivery system containing DOPC, DOPS, Chol, and $\mathrm{Ca}^{2+}$ for in vitro siRNA-mediated knockdown of genes in macrophages. Initial assessment of various transfection formulas revealed that DOPC/DOPS liposomes showed substantially higher knockdown efficiency of GFP in GFP-A549 cells than Lipofectamine2000 (Lipo2000), which is a popular and commercially available cationic liposomal transfection reagent (GFP knockdown efficiency of $76.84 \%$ by Ca-PS lipopolyplex compared to $49.21 \%$ by Lipo2000). In addition, the anionic DOPC/DOPS liposomes also showed much less cytotoxicity towards A549 cells than the cationic Lipo2000. We further optimized the PS-containing formula to have a molar ratio 4:3:3 for DOPC/DOPS/Chol, respectively. Together with $\mathrm{Ca}^{2+}$, this formula is referred to as Ca-PS lipopolyplex in this study. Our results revealed that, compared to Lipo2000,
Ca-PS lipopolyplex showed superior knockdown efficiency of both FADD and GAPDH genes in two types of macrophages, Ana-1, which is an immortalized mouse macrophage cell line, and BMDMs, which are freshly extracted primary cells. Knockdown efficiencies of FADD and GAPDH genes in macrophages were in the range of $63.7 \%-72.2 \%$ for $\mathrm{Ca}-\mathrm{PS}$ lipopolyplex compared to $28.1 \%-36.5 \%$ for Lipo 2000 . Knockdown of enhanced green fluorescent protein (EGFP) in BMDMs extracted from EGFP-transgenic mouse confirmed results from FADD/GAPDH experiments. Investigation of uptake efficiency of transfection complexes showed that macrophages assimilated markedly more transfection complexes in the case of Ca-PS lipopolyplex than Lipo2000, an effect directly attributed to PS. Study on cellular uptake and intracellular trafficking of siRNA showed that transfection by Ca-PS lipopolyplex gave rise to considerably increased cellular uptake in macrophages compared to Lipo2000, as well as lysosomal escape and localization to the perinuclear regions in BMDM. Lastly, investigations on macrophage polarization showed that transfection by both Ca-PS lipopolyplex and Lipo2000 carrying non-functional siRNAs (siNC) did not induce M1 or M2 polarization of macrophages. To summarize, Ca-PS lipopolyplex is an effective anionic liposomal formula possessing profoundly improved potency for siRNA-mediated knockdown of genes in macrophages. We believe that further research efforts can be devoted to the application of Ca-PS lipopolyplex in other immune cells such as T cells and DC cells.

\section{Material and methods \\ Animals}

Wild type and EGFP-transgenic C57BL/6 mice 6-8-weekold were purchased from the Model Animal Research Center of Nanjing University, China. All mice were housed under environmentally controlled conditions ( 12 hours of light/dark cycle at $22^{\circ} \mathrm{C}$ ).

\section{Cell cultures}

A549, 16HBE (HBE), and Ana-1 cells were purchased from the American Type Culture Collection (ATCC, Manassas, VA, USA). A549 and HBE cells were cultured in DMEM medium (Wisent, ST-BRUNO, Quebec, Canada) supplemented with $10 \%$ FBS (Gibco, Waltham, MA, USA) in a humidified incubator with $5 \% \mathrm{CO}_{2}$. Ana-1 cells were cultured in conditions identical to A549 and HBE cells except RMPI-1640 (Wisent, ST-BRUNO, Quebec, Canada) was used in place of DMEM. All cells were grown in the presence of $100 \mathrm{IU} / \mathrm{mL}$ penicillinstreptomycin (Invitrogen, Burlington, Canada). 
BMDMs were extracted using protocols described previously. ${ }^{27}$ Briefly, bone marrow cells were extracted from femurs of wild type and EGFP-transgenic C57BL/6 mice. Extracted cells were washed by PBS $(10 \mathrm{mM}, \mathrm{pH}=7.4)$ and treated with red blood cells lysis buffer (Solarbio, Beijing, China). The remaining cells were re-suspended in DMEM containing $20 \mathrm{ng} / \mathrm{mL}$ recombinant murine MCSF (PeproTech, Rocky Hill, NJ, USA), 10\% FBS, and $100 \mathrm{IU} / \mathrm{mL}$ penicillin-streptomycin (Invitrogen, Burlington, Canada). Purity of mature BMDMs was confirmed by flow cytometry on day seven post extraction.

\section{siRNAs and primers}

siRNAs used in this study are listed in Table S1. All siRNAs were synthesized by GenePharma, Shanghai, China. Primers used in this study are listed in Table S2. All primers were synthesized by Genescript, Nanjing, Jiangsu, China.

\section{Preparation and characterization of liposomes}

DOPC and DOPS were purchased from Avanti polar lipids (Alabaster, AL, USA). Chol was purchased from ThermoFisher Scientific (Waltham, MA, USA). Both types of liposomes (DOPC/DOPS and DOPC/DOPS/Chol) were prepared from a stock solution (compositions depending on the type of liposomes being prepared) containing $10 \mathrm{mg}$ of lipids dissolved in chloroform which was then allowed to dry and form a thin layer on the inner wall of a glass flask using a rotary evaporator. Molar ratios of the three types of liposomes were DOPC/DOPS=7:3; DOPC/DOPS/Chol=4:3:3. The lipid film was then dissolved in $2.5 \mathrm{~mL}$ of PBS buffer $(10 \mathrm{mM}$, $\mathrm{pH}=7.4$ ) followed by vortexing. The dissolved lipid solutions were sonicated and extruded through a $0.22 \mu \mathrm{m}$ syringe filter (Millipore, Darmstadt, Germany). The final concentration for all three types of liposomal solutions was $4 \mathrm{mg} / \mathrm{mL}$ and molar concentrations were calculated accordingly. Diameters and Zeta potentials of different liposomal solutions were measured in triplicate by Malvern Zetasizer Nano ZS90 (Malvern Instruments, Herrenberg, Germany).

\section{MST}

To determine the binding ability between liposomes and Annexin V, MST was performed as previously described. ${ }^{28}$ DOPC/DOPS and DOPC/DOPS/Chol liposomes were prepared as previously described in this study. Briefly, MST experiments were conducted in triplicate on a Monolith NT.115 system (NanoTemper Technologies) using hydrophilic capillaries (Nanotemper, K003 Monolith NT.115 Hydrophobic Capillaries). Annexin V-EGFP was re-suspended in the reaction buffer containing $10 \mathrm{mM}$ Hepes, $140 \mathrm{mM} \mathrm{NaCl}, \mathrm{pH} 7.4$, in the presence of $2.5 \mathrm{mM}$ $\mathrm{CaCl}_{2}$. For $\mathrm{K}_{\mathrm{d}}$ determinations, the concentration of Annexin V-EGFP was kept constant at $100 \mathrm{nM}$ and varying amounts of liposomes were titrated. Curve fitting and data analysis were done using Hill's equation by Graphpad Prism 6 .

\section{Transfection}

Cells were seeded into wells of 24-well plates so that a confluence of $70 \%-90 \%$ would be reached in 24 hours for transfection. Prior to transfection, the complete growing medium containing FBS was replaced by DMEM or RMPI-1640 containing the transfection complex depending on the cell type. For Lipo2000 and $\mathrm{CaCl}_{2}$ transfection, Lipo2000 or $\mathrm{CaCl}_{2}$ was mixed directly with appropriate siRNAs in DMEM or RPMI-1640. For PS-Ca lipopolyplex transfection, $\mathrm{CaCl}_{2}$ was first mixed with siRNAs to form $\mathrm{Ca}^{2+}$-siRNA carrying a net positive charge. PS-containing liposomes were then mixed with $\mathrm{Ca}^{2+}$-siRNA to form the Ca-PS lipopolyplex. Formulas and concentrations of reagents for transfection are listed in Table 1. Replacement of DMEM or RPMI-1640 by complete growth media was done at 6 hours post-transfection. Transfection efficiency,

Table I Transfection formulas used for screening

\begin{tabular}{|c|c|c|c|c|c|}
\hline $\begin{array}{l}\text { Transfection } \\
\text { ingredients }\end{array}$ & $\begin{array}{l}\text { Molar } \\
\text { ratio }\end{array}$ & $\begin{array}{l}\text { Stock solution } \\
\text { concentration }\end{array}$ & $\begin{array}{l}\text { siGFP } \\
\text { concentration (nM) }\end{array}$ & $\begin{array}{l}\text { Transfection } \\
\text { concentration }\end{array}$ & $\begin{array}{l}\mathrm{Ca}^{2+} \text { concentration } \\
(\mathrm{mM})\end{array}$ \\
\hline Lipo2000 & $\mathrm{N} / \mathrm{A}$ & $1 \mathrm{mg} / \mathrm{mL}$ & 40 & $\mathrm{I} \mu \mathrm{g} / \mathrm{mL}$ & N/A \\
\hline Lipo2000 & $\mathrm{N} / \mathrm{A}$ & $\mathrm{I} \mathrm{mg} / \mathrm{mL}$ & 40 & $2 \mu \mathrm{g} / \mathrm{mL}$ & N/A \\
\hline Lipo2000 & $N / A$ & $\mathrm{l} \mathrm{mg} / \mathrm{mL}$ & 40 & $4 \mu \mathrm{g} / \mathrm{mL}$ & $\mathrm{N} / \mathrm{A}$ \\
\hline DOPC/DOPS & $7: 3$ & $5.04 \mathrm{mM}$ & 40 & $10.08 \mu \mathrm{M}$ & 0.2 \\
\hline DOPC/DOPS & $7: 3$ & $5.04 \mathrm{mM}$ & 40 & $10.08 \mu \mathrm{M}$ & 1 \\
\hline DOPC/DOPS & $7: 3$ & $5.04 \mathrm{mM}$ & 40 & $10.08 \mu \mathrm{M}$ & 2 \\
\hline $\mathrm{CaCl}_{2}$ & $\mathrm{~N} / \mathrm{A}$ & I M & 40 & $10 \mathrm{mM}$ & $10 \mathrm{mM}$ \\
\hline $\mathrm{CaCl}_{2}$ & N/A & I M & 40 & $5 \mathrm{mM}$ & $5 \mathrm{mM}$ \\
\hline $\mathrm{CaCl}_{2}$ & N/A & I M & 40 & $2 \mathrm{mM}$ & $2 \mathrm{mM}$ \\
\hline
\end{tabular}


cytotoxicity and uptake efficiency were monitored at 48 hours post-transfection.

\section{Flow cytometry}

Flow cytometry was used in this study for multiple purposes. Green fluorescence of transfected cells was monitored by the FL1-H channel of the BD FACSCalibur flow cytometer (BD Biosciences, San Jose, CA, USA) to evaluate siRNAmediated knockdown of GFP or EGFP, and uptake efficiency of FAM-labeled transfection complexes. Flow cytometry was also employed to identify mature macrophages by F4/80 and CD11b (BD Biosciences, San Jose, CA, USA) after 7 days of differentiation, and M1 or M2 polarization of macrophages using CD11c and CD206 as markers. Purity of macrophages was calculated as percentage of cells positive for both F4/80 and CD11b.

Macrophages of M1 or M2 polarization were identified as CD11c+CD206- or CD11c-CD206+ cells, respectively. Positive controls, namely M1 and M2 polarized macrophages (Ana-1 and BMDM), were induced by LPS and IL-4, respectively.

\section{Cytotoxicity assay}

The viability of transfected cells was monitored using the CCK-8 kit (Beyotime, Nantong, China) according to the manufacturer's instructions. Briefly, $\sim 5 \times 10^{3}$ transfected cells of any given transfection treatment in this study were seeded into each well of 96-well plates. Samples were monitored at 48 hours post-transfection and in triplicate at $450 \mathrm{~nm}$ by the Tecan Infinite 200 PRO plate reader (Tecan Group Ltd., Männedorf, Switzerland). Cytotoxicity was calculated by normalizing OD450 values against untreated groups.

\section{RNA isolation and quantitative real-time PCR}

Total RNA of transfected cells was extracted using the Trizol reagent (Invitrogen, Carlsbad, CA, USA) according to the manufacturer's instructions. Reverse transcription was implemented using the reverse transcription kit (Toyobo, Kita-ku, Osaka, Japan). qPCR was performed using the AceQ qPCR SYBR Green Master Mix (Vazyme, Nanjing, China). The PCR cycling conditions were as follows: an initial start at $95^{\circ} \mathrm{C}$ for 10 minutes, followed by 40 cycles at $95^{\circ} \mathrm{C}$ for 15 seconds and $60^{\circ} \mathrm{C}$ for 1 minute.

\section{Western blot analysis}

Cells were washed twice with ice-cold PBS after collection. Total proteins in cells were lysed with RIPA buffer (protein inhibitor included) for 40 minutes and then centrifuged $\left(12,000 \mathrm{rpm}, 10\right.$ minutes, $\left.4^{\circ} \mathrm{C}\right)$. The extracted proteins were separated using 12\% SDS-PAGE and transferred to PVDF membranes (Millipore, Bedford, MA, USA). After that, the membranes were blocked in non-fat 5\% milk at room temperature for 1 hour and then blotted with an appropriate primary antibody at $4^{\circ} \mathrm{C}$ overnight. After three washes in PBS (10 mM, pH 7.4) containing $0.1 \%$ Tween 20 , the membranes were incubated in a second antibody of a mouse or rabbit, specific for each primary antibody, for 1 hour at room temperature the following day. After three washes in PBS with $0.1 \%$ Tween 20 , the blots were visualized using ECL detection reagents. Bands were quantified by the Image J software.

\section{Fluorescence microscopy}

For microscopic observation on cellular uptake of siRNA, FAM-siRNA was used as donor for green fluorescence. Hoechst 33,342 (KeyGen BioTECH, Nanjing, Jiangsu, China) and DiI (KeyGen BioTECH, Nanjing, Jiangsu, China) were used to stain the nuclei and the membrane of cells and as donors for blue and red fluorescence, respectively. Cells at 6 hours post-transfection (transfected by either Ca-PS lipopolyplex or Lipo2000) were imaged by a Zeiss Axio Vert.A1microscope equipped with an Olympus X-cite 120 Q light source.

For microscopic observation on intracellular trafficking of siRNA, FAM-siRNA was used as donor for green fluorescence. Hoechst 33,342 and LysoTracker-Red (KeyGen BioTECH, Nanjing, Jiangsu, China) were used to stain the nuclei and lysosomes of BMDMs and as donors for blue and red fluorescence, respectively. BMDMs at 1, 6, 12, 24 hours post-transfection (transfected by either Ca-PS lipopolyplex or Lipo2000) were imaged by a Zeiss Axio Vert.A1microscope equipped with an Olympus X-cite 120 Q light source.

\section{Statistical analyses}

Data in this study are presented as mean \pm SD. Student's $t$-test was used for statistical analysis. All statistical analyses were conducted using Graphpad Prism 6. $P$-value less than 0.05, $0.01,0.001$, and 0.0001 is indicated by $*, * *, * * *$, and $* * * *$, respectively.

\section{Results}

\section{Screening for ideal PS-containing formula for siRNA-mediated knockdown transfection}

In the process of screening for ideal PS-containing liposomal formula, we produced negatively charged liposomes by mixing neutral lipid DOPC and negatively charged DOPS. 
However, the DOPS/DOPC liposomes require reversal of surface charge in order to bind nucleotides carrying negative charges; therefore, calcium ions were used as a third component of this transfection formula. The formula screening process was implemented based on quantification of siRNA-mediated knockdown of GFP fluorescence in GFP-A549 cells, a straightforward and quantifiable way to evaluate knockdown efficiency. GFP fluorescence reduction was monitored by flow cytometry. Table 2 summarizes all formulas used for the screening process including a control, Lipo2000. Bar graphs showing statistical significance of data in Table 2 are shown in Figure S1.

We monitored both knockdown efficiency and cytotoxicity for each formula. Results are displayed in Figure 1. Figure 1A shows four fluorescence microscopy images of differentially treated GFP-A549 cells. Cells treated with Lipo2000 plus siNC showed no reduction of fluorescence compared to the untreated group (image not shown). $\mathrm{CaCl}_{2}$ at $10 \mathrm{mM}$ showed negligible knockdown of GFP. Lipo2000 at $2 \mu \mathrm{g} / \mathrm{mL}$ delivered significant knockdown of GFP fluorescence from siGFP. However, DOPC/DOPS at $10.08 \mu \mathrm{M}$ combined with $2 \mathrm{mM} \mathrm{Ca}^{2+}$ showed observably more potent knockdown efficiency than both $\mathrm{CaCl}_{2}$ and Lipo2000 treatments. Figure 1B shows the histogram of GFP fluorescence of representative treatments derived from flow cytometry. Again, DOPC/DOPS at $10.88 \mu \mathrm{M}$ combined with $2 \mathrm{mM}$ $\mathrm{Ca}^{2+}$ showed the highest level of GFP knockdown compared to the siNC groups. Lipo2000 at $2 \mu \mathrm{g} / \mathrm{mL}$ achieved significant knockdown of GFP fluorescence, but its potency was vastly outcompeted by the DOPC/DOPS/Ca ${ }^{2+}$ treatment. Surprisingly, $\mathrm{Ca}^{2+}$ alone in the form of $\mathrm{CaCl}_{2}$ at all assayed concentrations didn't show obvious knockdown effects (knockdown efficiency less than 10\%). Transfection using calcium chloride is a well-established method. ${ }^{29,30}$ Nevertheless, different cells may respond differently to the calcium-based method and no reports on transfection of A549 using calcium phosphate have been published. Therefore, it is hypothesized that calcium-based transfection is not an appropriate protocol for A549. However, the ineffectiveness of $\mathrm{Ca}^{2+}$ alone as a transfection reagent in our assay confirms that the superior knockdown efficiency of the DOPC/DOPS/ $\mathrm{Ca}^{2+}$ combination is indeed a contribution from PS, instead of $\mathrm{Ca}^{2+}$. Figure $1 \mathrm{C}$ shows the cytotoxicity of each single formula. As expected, the negatively charged DOPC/DOPS liposomes (last three columns) displayed much less cytotoxicity than positively charged Lipo2000 and $\mathrm{CaCl}_{2}$. All three DOPC/DOPS treatments showed cell viability higher than $80 \%$ while other treatments dropped below $80 \%$ (except for the siRNA group, which is a control group). In fact, high cytotoxicity of positively charged liposomes has been reported many times. Figure 1D shows the knockdown efficiency of every single treatment listed in Table 1. Knockdown efficiency is shown as normalized GFP fluorescence (obtained as mean fluorescence from flow cytometry) of each siGFP group against its respective siNC group. Consistent with previous results, DOPC/DOPS/ $\mathrm{Ca}^{2+}$ treatments showed superior knockdown efficiency. Treatment with DOPC/DOPS and $2 \mathrm{mM} \mathrm{Ca}^{2+}$ showed a GFP knockdown efficiency of $76.84 \% \pm 1.4 \%$, the highest among the DOPC/DOPS groups. This efficiency is higher than $49.21 \% \pm 3.6 \%$ achieved by $2 \mu \mathrm{g} / \mathrm{mL}$ Lipo 2000 which was the highest among the Lipo2000 groups. Therefore, the ideal formula for the PS-containing liposome is $10.08 \mu \mathrm{M}$ DOPC/DOPS plus $2 \mathrm{mM} \mathrm{Ca}^{2+}$, as judged from the screening process using knockdown efficiency of GFP fluorescence in GFP-A549 as a standard.

\section{Optimization and biophysical properties of the DOPC/DOPS liposomes}

We further optimized the formula of the DOPC/DOPS liposomes using cholesterol as a stabilizer. Cholesterol could reduce the rate of oxidation, improve membrane

Table 2 DLS parameters

\begin{tabular}{l|l|l|l}
\hline Formula & Diameter $(\mathbf{n m})$ & Zeta potential $(\mathbf{m V})$ & PDI \\
\hline DOPC:DOPS=7:3 & $101.2 \pm 0.4$ & $-42.1 \pm 2.9$ & $0.19 \pm 0.004$ \\
DOPC:DOPS:Chol=4:3:3 & $109.6 \pm 2.1$ & $-33.6 \pm 1.2$ & $0.19 \pm 0.004$ \\
DOPC:DOPS:Chol=4:3:3 & $117.3 \pm 4.3$ & $-28.7 \pm 2.1$ & $0.21 \pm 0.007$ \\
I2-month storage at 4 ${ }^{\circ} \mathrm{C}$ & & & \\
DOPC/DOPS+Ca ${ }^{2+}+$ siRNA $^{2+}$ & $536.6 \pm 7.7$ & $+7.8 \pm 1.1$ & $0.40 \pm 0.015$ \\
$\mathrm{Ca}^{2+}+$ siRNA & $283.8 \pm 5.4$ & $+37.7 \pm 2.4$ & $0.26 \pm 0.009$ \\
DOPC/DOPS/Chol+Ca ${ }^{2+}+$ siRNA & $519.1 \pm 4.5$ & $+7.3 \pm 1.6$ & $0.22 \pm 0.007$ \\
Lipo2000 $_{\text {Lipo2000+siRNA }}$ & $106.6 \pm 1.7$ & $+34.6 \pm 0.9$ & $0.17 \pm 0.003$ \\
\hline
\end{tabular}


A

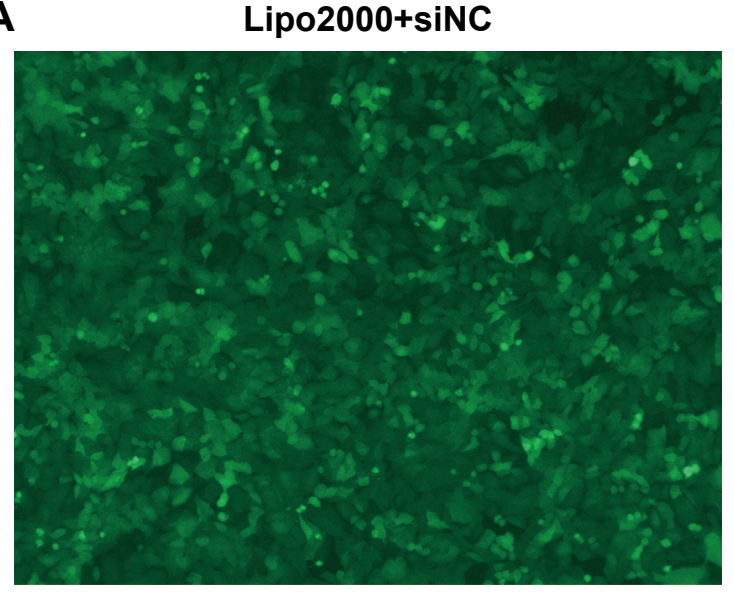

Lipo2000 $2 \mu \mathrm{g} / \mathrm{mL}+$ siGFP
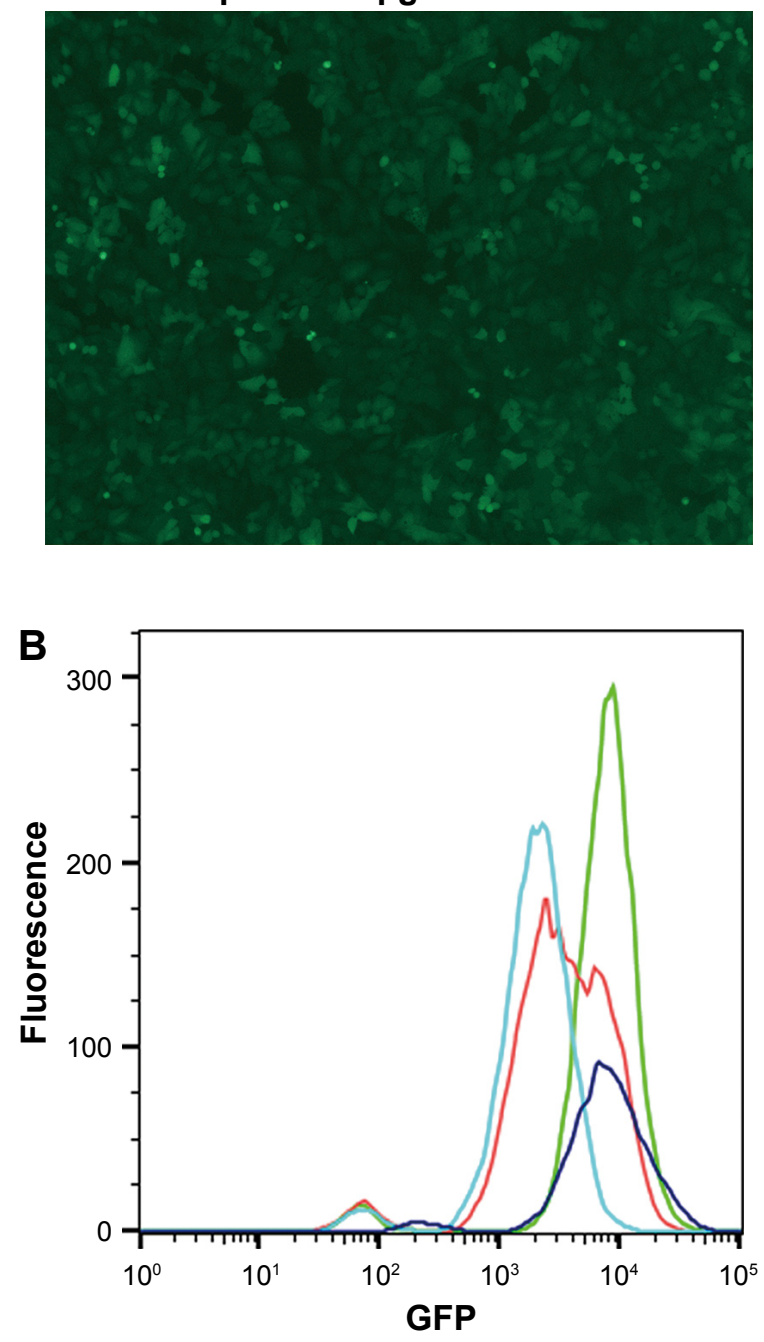

- Lipo2000 $2 \mu \mathrm{g} / \mathrm{mL}+\mathrm{siNC}$ Lipo2000 $2 \mu \mathrm{g} / \mathrm{mL}+$ siGFP $\mathrm{CaCl}_{2} 10 \mathrm{mM}+\mathrm{siGFP}$ DOPC/DOPS $10.88 \mu \mathrm{M}+$ siGFP
$\mathrm{CaCl}_{2} 10 \mathrm{mM}+$ siGFP

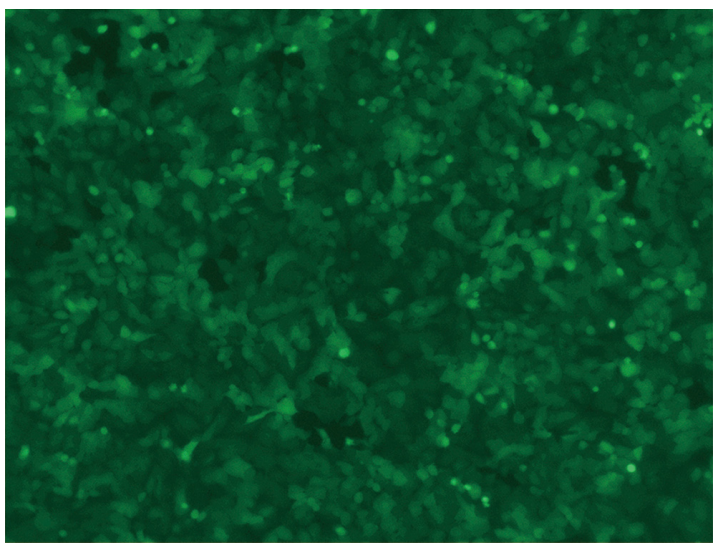

DOPC/DOPS $10.88 \mu \mathrm{M}+$ siGFP

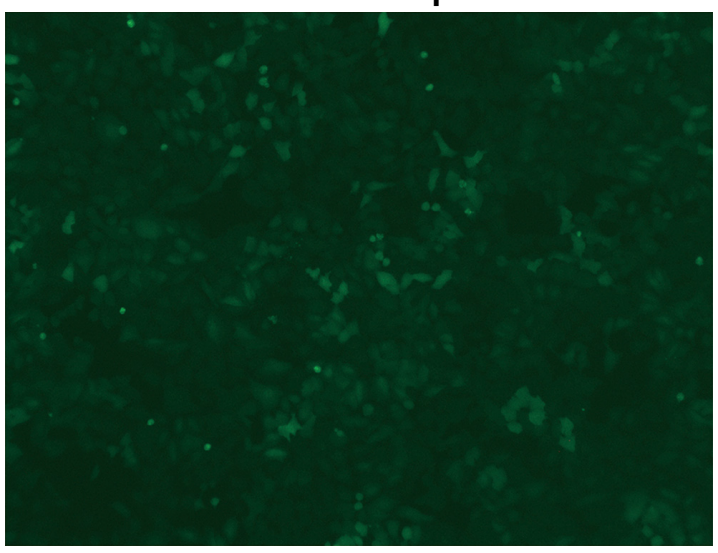

C

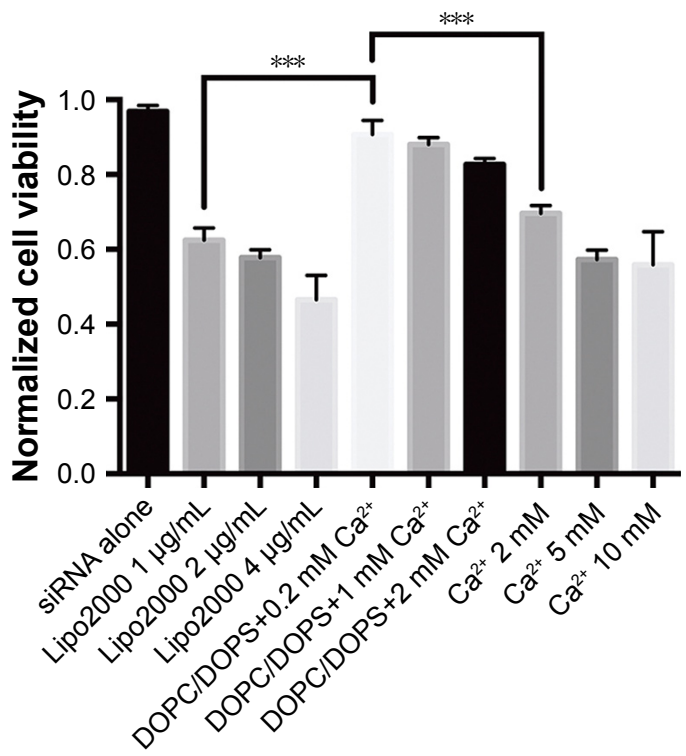

Figure I (Continued) 


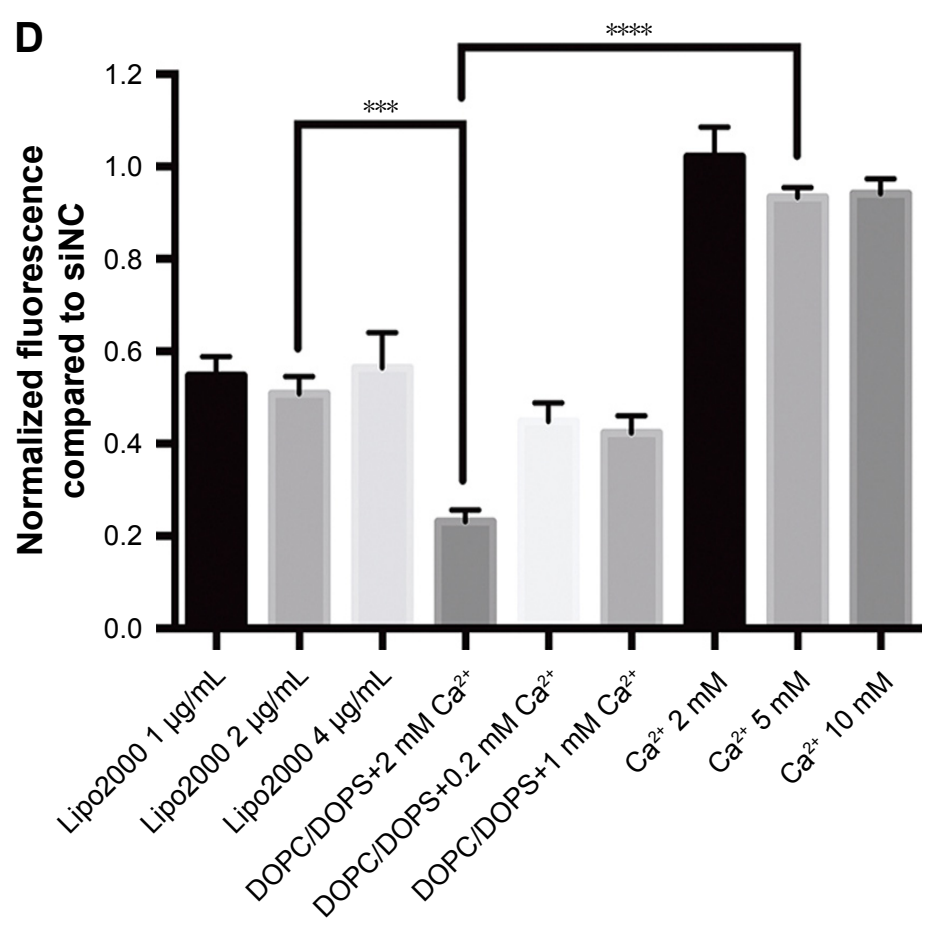

Figure I Screening for ideal PS-containing transfection formula using siGFP-mediated knockdown assay of GFP-A549 cells.

Notes: (A) Fluorescence microscopy of transfected GFP-A549 cells. GFP-A549 cells were treated with different transfection formulas for 48 hours. GFP knockdown was assessed by observing GFP fluorescence intensity using a fluorescence microscope. Treatment is indicated on top of each image. (B) GFP histogram of transfected GFP-A549 cells. GFP-A549 cells transfected for 48 hours were harvested and monitored for GFP intensity using flow cytometry. Treatments are indicated on the upper left corner of this graph. (C) Cytotoxicity of different transfection formulas. CCK-8 assay was used to assess cytotoxicity of each transfection formula within a 48 -hour period. (D) GFP knockdown efficiency of different transfection formulas. GFP intensity data were compiled and analyzed from flow cytometry. GFP knockdown efficiency is displayed as the ratio between GFP fluorescence intensity of each treatment group and that of its respective siNC control group. $* * * P<0.00 \mathrm{I}$, and $* * * * P<0.000 \mathrm{I}$.

permeability and increase resistance to aggregation of unsaturated DOPS/DOPC liposomes..$^{31-33} \mathrm{We}$ formulated a new PS-containing liposomes composed of DOPC/ DOPS/Chol at a molar ratio of 4:3:3. The molar ratio of cholesterol used in this study, namely $30 \%$, has been used in several published reports. ${ }^{34,35}$ Biophysical properties were then probed for liposomes of various compositions. Table 2 lists parameters measured by DLS. It is evident from Table 3 that both types of PS-containing liposomes (row 1 and 2) have a size similar to Lipo2000 (row 5), although DOPC/DOPS/Chol liposome is slightly larger than its counterpart. However, assembly of both types of PS-containing liposomes with $\mathrm{Ca}^{2+}$ and siRNA gives rise to a much increased particle diameter (both greater than $500 \mathrm{~nm})$ compared to Lipo2000 (176.6 nm). Zeta potential values indicate that both types of PS-containing liposomes are fairly stable and have a net negative charge irrespective of the addition of the neutral cholesterol. On the other hand, the commercially available and widely used Lipo2000 has a Zeta potential of $+34.6 \pm 0.9 \mathrm{mV}$, whose absolute value is very close to the DOPC/DOPS/Chol liposome (34.6 vs 33.6). Lastly, the DOPC/DOPS/Chol liposomes exhibited slightly increased size and decreased Zeta potential after a 12-month storage at $4^{\circ} \mathrm{C}$. Nevertheless, further study showed that DOPC/DOPS/Chol liposomes maintained high transfection potency and liposomal integrity after a 1 -year storage period at $4^{\circ} \mathrm{C}$, the results of which will be presented in the next section. These results demonstrate that DOPC/DOPS/Chol liposomes are very similar to Lipo2000 in terms of DLS parameters despite their opposite charges, and therefore, are expected to be suitable for long-term application of transfection.

Table 3 Knockdown efficiencies of FADD and GAPDH genes in Ana-I and BMDM cells calculated based on normalized protein levels (Western blot)

\begin{tabular}{l|l|l|l|l}
\hline Transfection reagents & Ana-I FADD & BMDM FADD & Ana-I GAPDH & BMDM FADD \\
\hline Ca-PS lipopolyplex & $64.7 \% \pm 5.9 \%$ & $64.8 \% \pm 3.9 \%$ & $72.2 \% \pm 3.1 \%$ & $63.7 \% \pm 5.9 \%$ \\
Lipo2000 & $32.5 \% \pm 4.2 \%$ & $36.5 \% \pm 3.5 \%$ & $28.1 \% \pm 10.2 \%$ \\
\hline
\end{tabular}


We designed the PS-containing liposomes for transfection of the hard-to-transfect macrophages due to the hypothesis that PS can effect a much more effective uptake of liposomes, compared to routine transfection reagents, by macrophages in a way similar to apoptotic bodies being engulfed by phagocytes (such as macrophages). This is because apoptotic bodies derived from apoptotic cells can attract macrophages to their proximity and trigger phagocytosis, and both events rely heavily on PS presented on the surface of apoBDs. The PS component of the DOPC/DOPS/Chol liposomes was then examined using affinity analysis by MST. The purpose of this experiment was to reveal whether DOPS contained within liposomes is functional, that is whether it can bind the classic Annexin V, a protein capable of binding PS and widely used for apoptosis detection. Figure 2 shows the dosedependent change of fluorescence of Annexin V-EGFP upon binding DOPC/DOPS/Chol liposomes or DOPC liposomes of varying concentrations. The $\mathrm{R}^{2}$ value measuring the quality of the fit is 0.9898 for DOPC/DOPS/Chol liposomes and the $\mathrm{K}_{\mathrm{d}}$ value is $12.97 \mu \mathrm{M}$. These results suggest that PS of the DOPC/DOPS/Chol liposomes are capable of Annexin $\mathrm{V}$ binding and therefore remain functional in liposomal content. We also monitored the stability and transfection potency of two types of PS-containing liposomes over time. By direct observation of liposomal solutions, we realized that DOPC/DOPS liposomes without cholesterol began to aggregate and form precipitates after only 1 month of storage at $4^{\circ} \mathrm{C}$ while the DOPC/DOPS/Chol liposomal solution remained clear for more than a year. The siGFP-mediated knockdown assay of GFP-A549 cells showed that the DOPC/

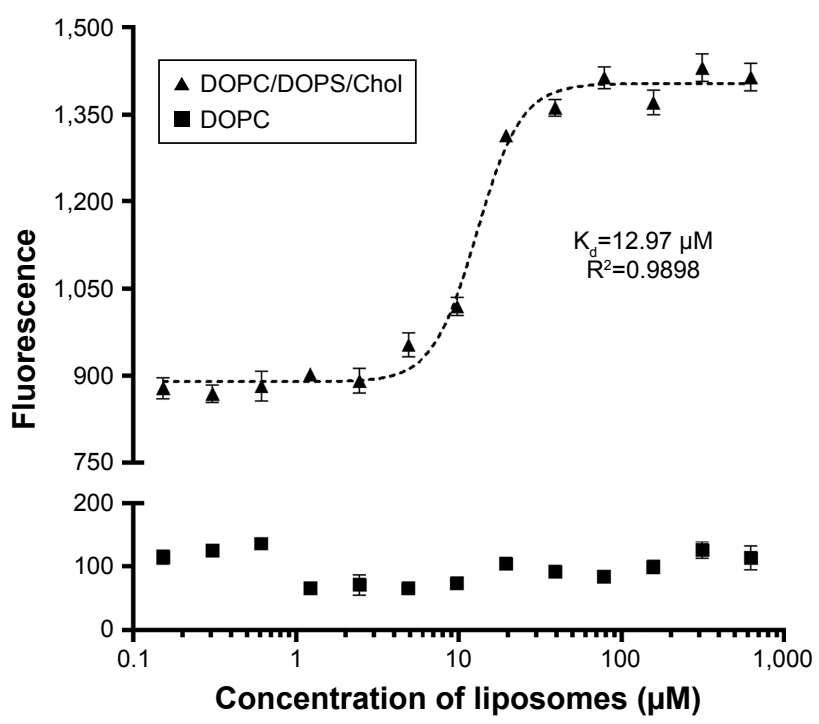

Figure 2 PS-Annexin $\mathrm{V}$ binding affinity analysis using DOPC/DOPS/Chol and DOPC liposomes as PS donors.
DOPS/Chol liposome is slightly less efficient than DOPC/ DOPS liposomes, whereas its potency did not decline over a one-year storage period (Figure 3 ). On the other hand, DOPC/DOPS liposomes became much less efficient and far more cytotoxic after a storage period of 3 month (Figure 2). Therefore, DOPC/DOPS/Chol at molar ratio 4:3:3 is an ideal PS-containing liposomal formula and is hereafter referred to as Ca-PS lipopolyplex.

\section{Assessment of Ca-PS lipopolyplex by siRNA-} mediated knockdown of genes in multiple cells Ca-PS lipopolyplex was then used in multiple cells, including primary cells and cell lines, to evaluate its potency in siRNA-mediated knockdown of specific genes. In this part, Ca-PS lipopolyplex was used at a concentration of $10.08 \mu \mathrm{M}$ in combination with $2 \mathrm{mM} \mathrm{Ca}^{2+}$ and $40 \mathrm{nM}$ siRNA, a transfection formula proven to be most efficient among all formulas assayed in the previous session. Lipo2000 was used as a control at a concentration of $2 \mu \mathrm{g} / \mathrm{mL}$ with $40 \mathrm{nM}$ siRNA for comparison purposes. The cells used are non-fluorescent NSCLC cell line A549, immortalized bronchus epithelial cell line HBE, mouse macrophage cell line Ana-1 and mouse primary BMDM. We attempted to knockdown FADD, a

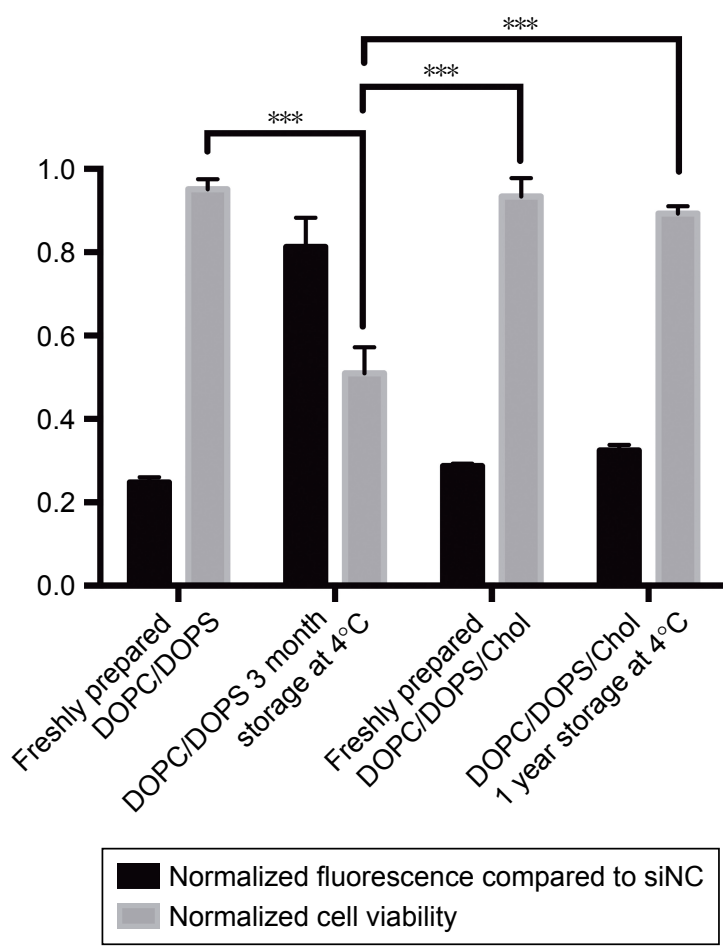

Figure 3 GFP knockdown efficiency and cytotoxicity of PS-containing liposomal formulas at various storage time points.

Notes: GFP knockdown efficiency was evaluated using flow cytometry and cytotoxicity was evaluated using CCK-8 assay over a 48-hour transfection period. The y-axis represents either normalized fluorescence or normalized cytotoxicity. ${ }^{* * * P}<0.00 \mathrm{I}$. 
well-known adaptor in apoptosis, and GAPDH, a housekeeping enzyme involved in glycolysis. Results are shown in Figure 4. Figure 4A shows mRNA levels of FADD and GAPDH detected by qPCR in four types of cells 48 hours after transfection by various siRNA treatments. Figure $4 \mathrm{~B}$ shows protein levels of FADD and GAPDH detected by Western blot in four types of cells 48 hours after transfection by various siRNA treatments. In both Figure 4A and B, mRNA and protein levels for each treatment group, including the untreated and siNC groups, are displayed and shown as fold change of the target gene compared to the internal control Actin. For Western blot results, protein levels were quantitated by the ImageJ software and are shown beneath the blot images as bar graphs. Knockdown efficiencies at which mRNA and proteins levels of the siFADD or siGAPDH groups were normalized against their respective siNC groups are shown in Figure 4C. Knockdown efficiency of the FADD gene by Ca-PS lipopolyplex is significantly higher than that by Lipo2000 in all four types of cells as shown in Figure 4C. Nevertheless, it is noteworthy that the superiority of Ca-PS lipopolyplex over Lipo2000 is much more profound in Ana-1 and BMDM cells than in A549 and HBE cells. It is hypothesized that the PS component is pivotal to enhanced knockdown efficiency in macrophages by the Ca-PS lipopolyplex. However, Figure 4C also shows that knockdown efficiencies of GAPDH in A549 and HBE cells by Ca-PS lipopolyplex and Lipo2000 are statistically indiscernible. In contrast, Ca-PS lipopolyplex is conspicuously more efficient than Lipo2000 in knocking down GAPDH in macrophages (Ana-1 and BMDM). Therefore, Ca-PS lipopolyplex is a more effective transfection reagent for siRNA-mediated knockdown in macrophages than Lipo2000. Knockdown efficiencies of FADD and GAPDH genes in Ana-1 and BMDM are listed in Table 3.

We then employed a more direct way of observing knockdown efficiency in macrophages. We extracted BMDMs from transgenic mouse carrying a universal EGFP gene. In this case, the extracted BMDM becomes fluorescent and can be studied in a way identical to that of GFP-A549 cells previously used in this research (Figure 5). Figure 5A shows both bright field and fluorescence images of extracted EGFPBMDMs. Figure 5B shows the fluorescence images of transfected EGFP-BMDM cells. Obvious diminishing of EGFP fluorescence was observed in Ca-PS lipopolyplex-treated cells while only slight reduction was observed in Lipo2000treated cells. Figure 5B and C show quantitated EGFP fluorescence by flow cytometry. Again, Ca-PS lipopolyplex delivered significant EGFP repression while Lipo2000 only delivered marginal knockdown effects, a knockdown efficiency of $59.7 \% \pm 1.8 \%$ by Ca-PS lipopolyplex compared to $21.9 \% \pm 3.4 \%$ by Lipo 2000 .

\section{Uptake efficiency and intracellular trafficking of siRNA delivered by Ca-PS lipopolyplex}

In this part, we attempted to investigate the effects of PS on the uptake of transfection complexes (Ca-PS lipopolyplex+siRNA or Lipo $2000+$ siRNA) by macrophages. We investigated whether the enhanced knockdown efficiency of macrophages by Ca-PS lipopolyplex can be attributed to its PS and therefore PS-mediated phagocytosis by macrophages. We used FAMlabeled siRNA to quantify siRNA uptake by monitoring FAM fluorescence of various cells treated by FAM-labeled transfection complexes at different time points. Figure $6 \mathrm{~A}$ shows the FAM fluorescence histograms monitored and generated by flow cytometry 6 hours post-transfection. It is obvious that both types of transfection complexes (Ca-PS lipopolyplex+siRNA or Lipo2000+siRNA) delivered considerable FAM fluorescence to recipient cells compared to the untreated and FAM-siRNA groups in A549 and HBE cells at 6 hours post-transfection, indicating that $\mathrm{Ca}-\mathrm{PS}$ lipopolyplex and Lipo2000 are equivalent in their ability to transfect and deliver siRNAs to A549 and HBE cells. However, Ca-PS lipopolyplex delivered prominently more fluorescence to two types of macrophages than Lipo2000, which indicates that Ca-PS lipopolyplex is more effective in delivering siRNAs to macrophages. Figure $6 \mathrm{~B}$ shows that fluorescence of the recipient cells, regardless of the cell type, declined over time (6-60 hour). It is notable that in A549 and HBE cells, the initial fluorescence and the rate of declination are almost identical for both types of transfection particles. However, in two types of macrophages, both initial fluorescence and rate of declination are different. For both Ana-1 and BMDM cells, Ca-PS lipopolyplex delivered much more FAM fluorescence than Lipo2000 at 6 hours post-transfection, whereas FAM fluorescence dropped steeply within the time interval of 6-24 hour post-transfection. On the other hand, Lipo2000 was only able to deliver less than one third of FAM fluorescence compared to Ca-PS lipopolyplex in macrophages, although the rate of diminishing of fluorescence appeared to be slower. To summarize, we monitored the ability of Ca-PS lipopolyplex and Lipo2000 to deliver siRNA to recipient cells. Our results showed that in non-macrophage cells such as A549 and HBE, they are equally capable. In the case of macrophages, PS renders Ca-PS lipopolyplex much more 


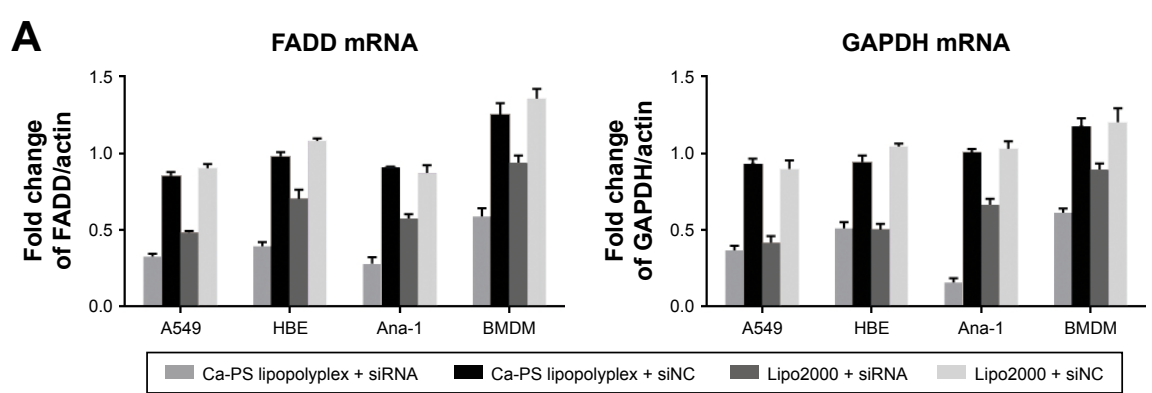

B

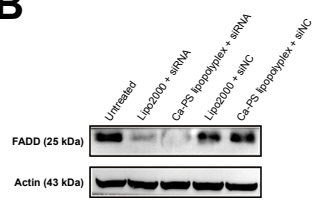

FADD A549

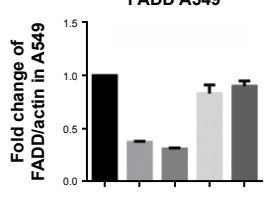

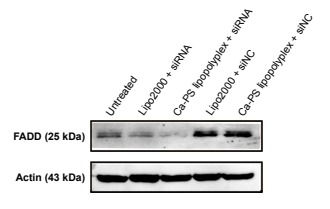

FADD HBE
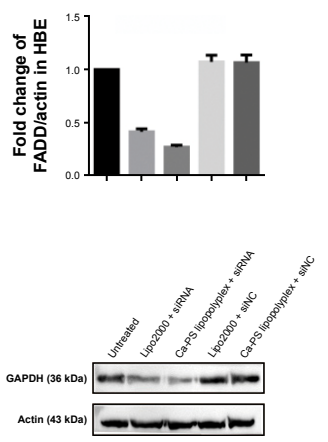

GAPDH HBE

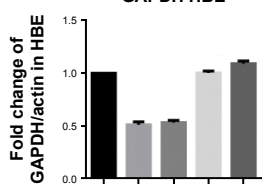

FADD knockdown efficiency (mRNA level)

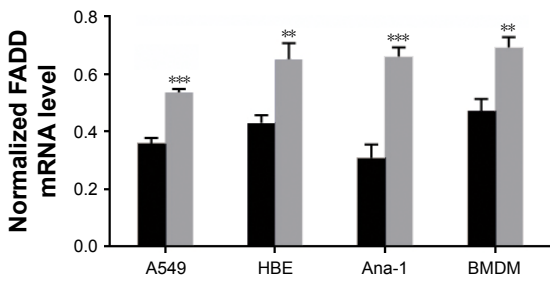

FADD knockdown efficiency (protein level)

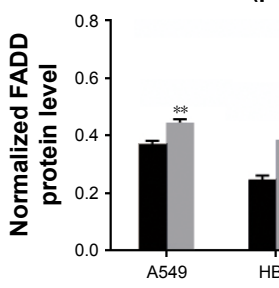

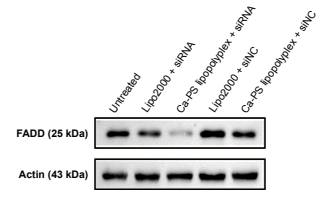

FADD Ana-1
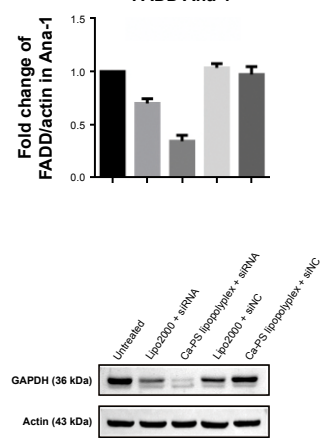

GAPDH Ana-1

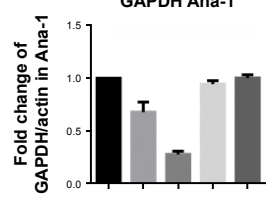

GAPDH knockdown efficiency (mRNA level)

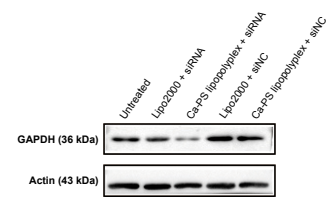

GAPDH BMDM
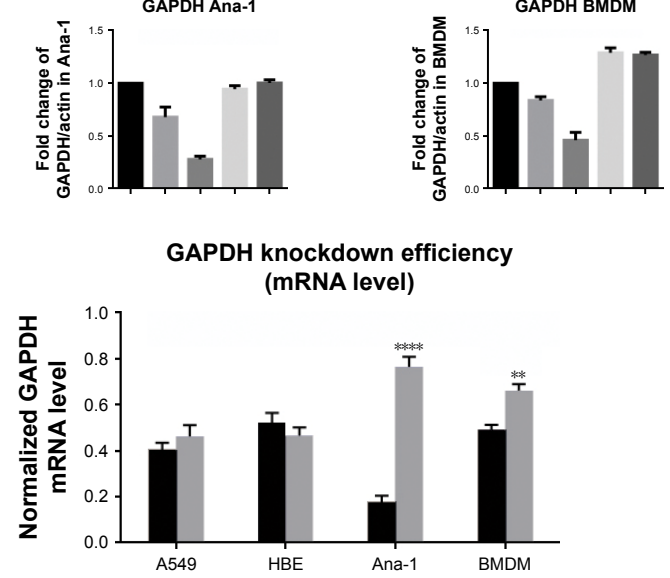

GAPDH knockdown efficiency (protein level)

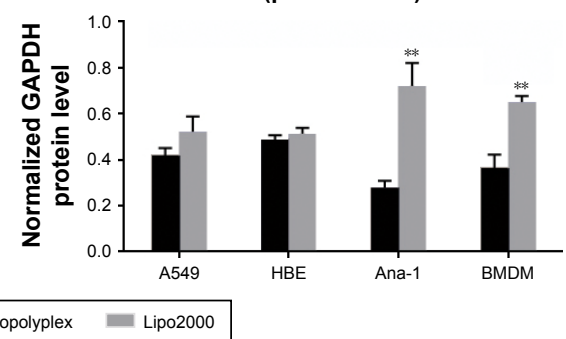

Figure 4 Knockdown efficiency of Ca-PS lipopolyplex in multiple cells.

Notes: A549, HBE, Ana-I and BMDM cells were treated by siRNA-mediated knockdown delivered by Ca-PS lipopolyplex or Lipo2000. Knockdown efficiency was evaluated by probing changes in mRNA and protein levels of siRNA-targeted genes. Each individual graph is associated with a title. (A) mRNA levels of FADD and GAPDH in four types of cells 48 hours post various transfection treatments. (B) Protein levels of FADD and GAPDH in four types of cells 48 hours post various transfection treatments. (C) Knockdown efficiency of FADD and GAPDH presented as normalized mRNA level or protein level. $* * P<0.01$, $* * * P<0.001$, and $* * * * P<0.000$ I. 
A

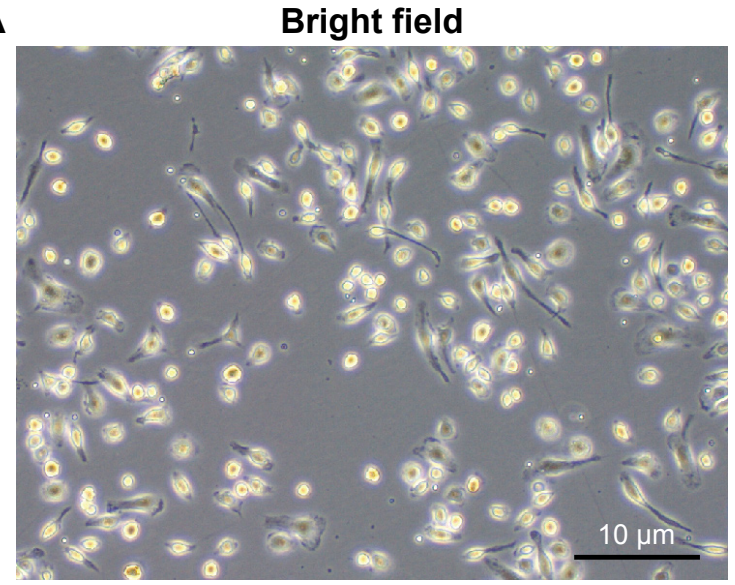

B

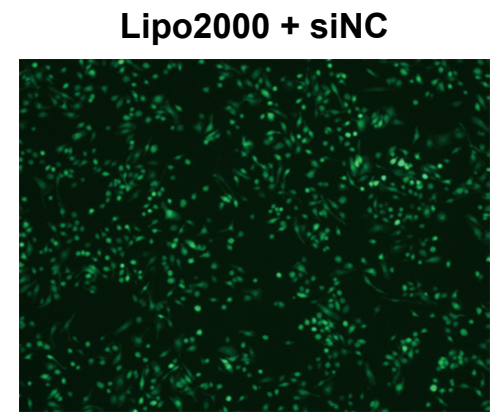

C

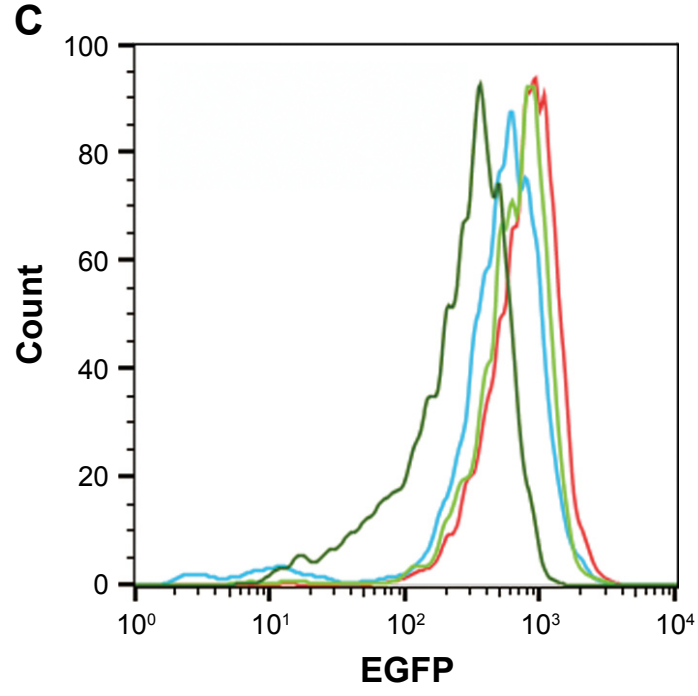

- Ca-PS lipopolyplex+siRNA

- Lipo2000+siRNA

- Ca-PS lipopolyplex+siNC

- Lipo2000+siNC

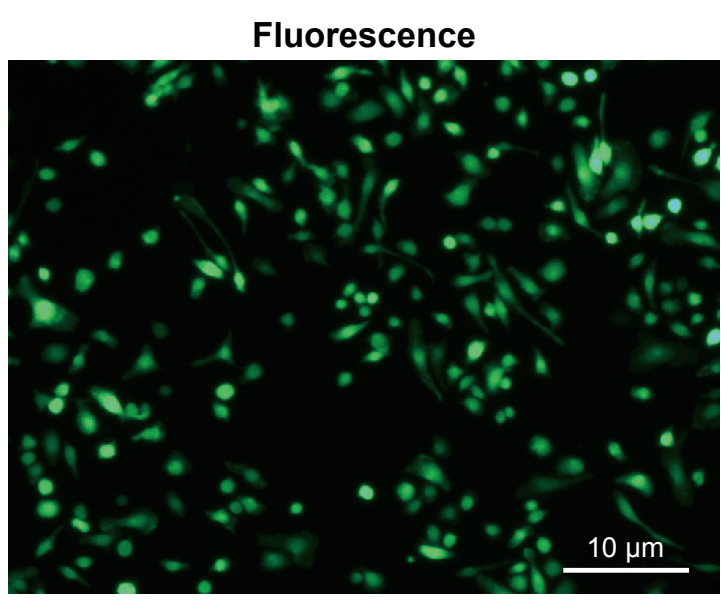

Ca-PS lipopolyplex + SiEGFP

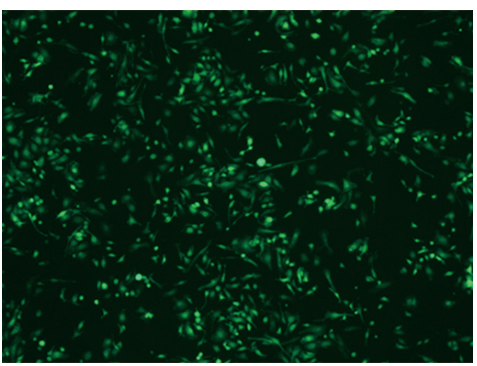

D

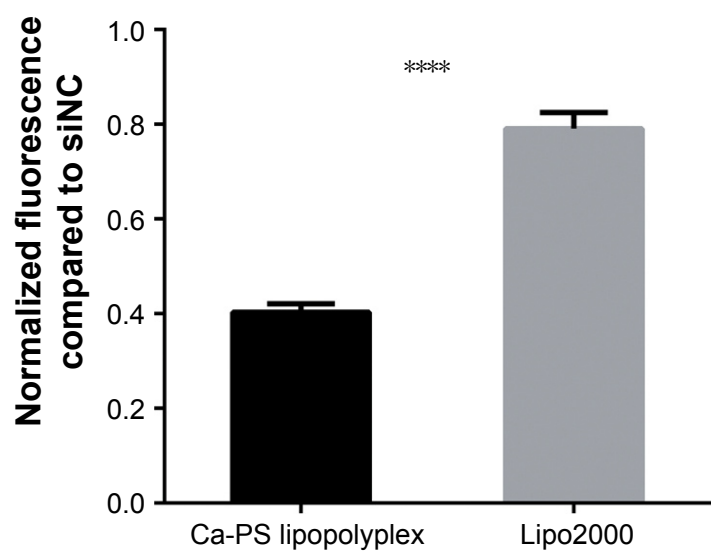

Figure 5 Knockdown of EGFP in EGFP-BMDMs by Ca-PS lipopolyplex.

Notes: (A) Bright field and fluorescence images of extracted BMDM of the same microscopic field. (B) Fluorescence microscopy of EGFP-BMDMs treated by Ca-PS lipopolyplex or Lipo2000 carrying siEGFP. Treatment is indicated on top of each graph. (C) EGFP histogram of transfected EGFP-BMDMs. EGFP-BMDM cells transfected for 48 hours were harvested and monitored for EGFP intensity using flow cytometry. Treatments are indicated on the upper left corner of this graph. (D) Comparison of EGFP knockdown efficiencies in EGFP-BMDMs by Lipo2000 and Ca-PS lipopolyplex. ***** $P<0.000$ I. 
effective than Lipo2000 in terms of delivering siRNA to recipient cells.

We further characterized siRNA uptake in these types of cells by FAM-siRNA and fluorescence microscopy. Cells undergoing siRNA transfection were imaged at 6 hours post-transfection. Figure 7 shows fluorescence images of four types of cells labeled by two fluorescence dyes, a nuclear dye Hoechst 33,342 and a membrane dye DiI. For A549 and HBE cells, siRNA uptake per cell at 6 hours post-transfection was similar between Ca-PS lipopolyplex and Lipo2000 treatments. It is noteworthy that transfection by Ca-PS lipopolyplex led to a slightly more clustered distribution of siRNAs while Lipo2000 led to a more homogenous siRNA distribution in A549 and HBE cells. This may be ascribed to the larger size of the Ca-PS/siRNA transfection complex. On the other hand, transfection by Ca-PS lipopolyplex achieved markedly higher siRNA uptake than Lipo2000 in two types of macrophages, namely Ana-1 and BMDM. Moreover, treatment by Lipo2000 resulted in rounding of BMDMs (BMDMs became spindle-shaped upon MCSF activation, before which they appeared as circle-shaped similar to Ana-1), which may be due to the higher cytotoxicity of Lipo2000.
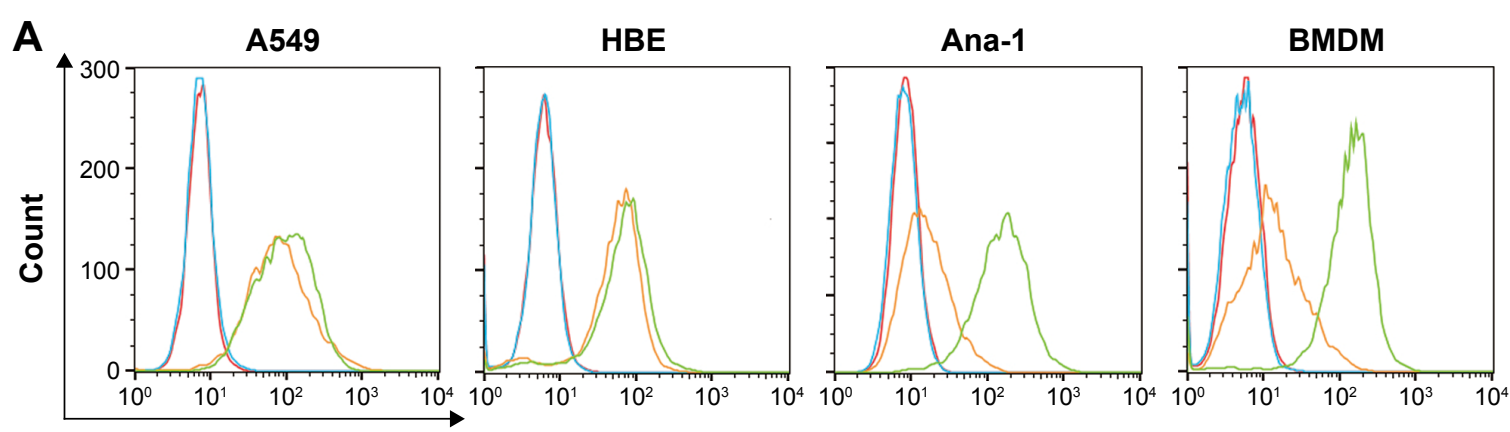

FAM-siRNA fluorescence

\begin{tabular}{|c|c|c|c|}
\hline Untreated & FAM-siRNA alone & Lipo2000+FAM-siRNA & Ca-PS lipopolyplex+FAM-siRNA \\
\hline
\end{tabular}

B

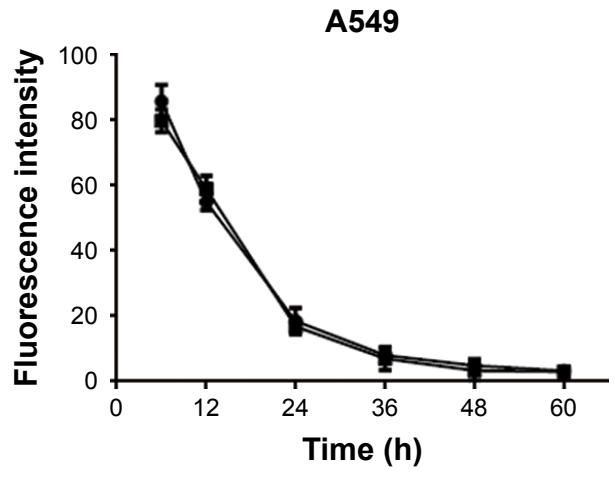

Ana-1

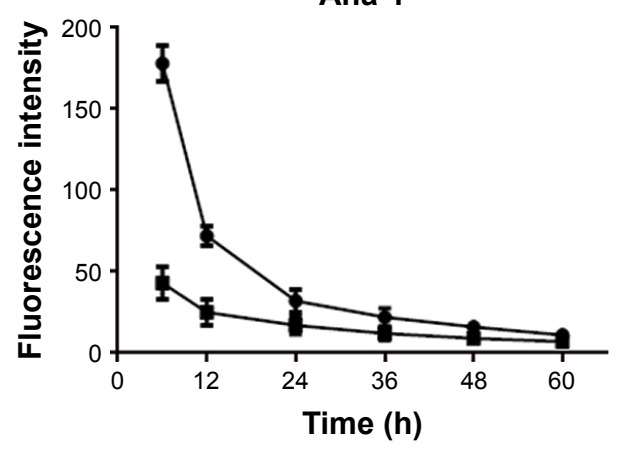

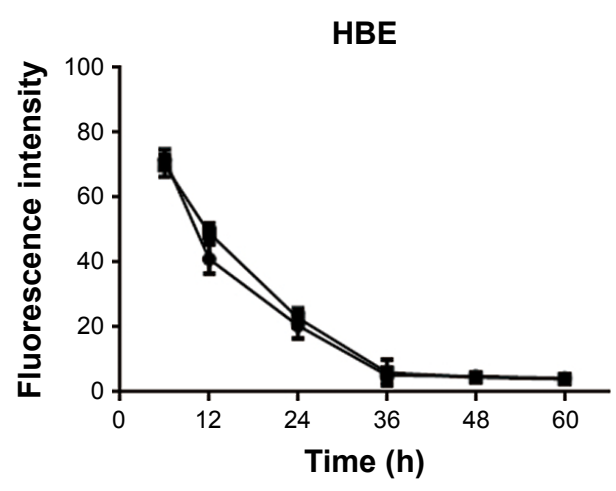

BMDM

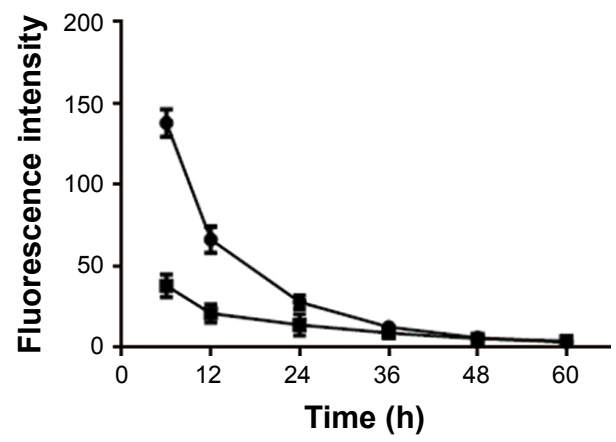

Time (h)

Ca-PS lipopolyplex

Lipo2000

Figure 6 Monitoring the uptake efficiency of transfection complexes by transfected cells. Uptake efficiency was evaluated using FAM-labeled siRNA transfection complex at 6-60 hour post-transfection by flow cytometry.

Notes: (A) Fluorescence histograms generated by flow cytometry 6 hours post-transfection of four types of cells. (B) Time-dependent declination of fluorescence from 6 to 60 hour post-transfection of four types of cells. 


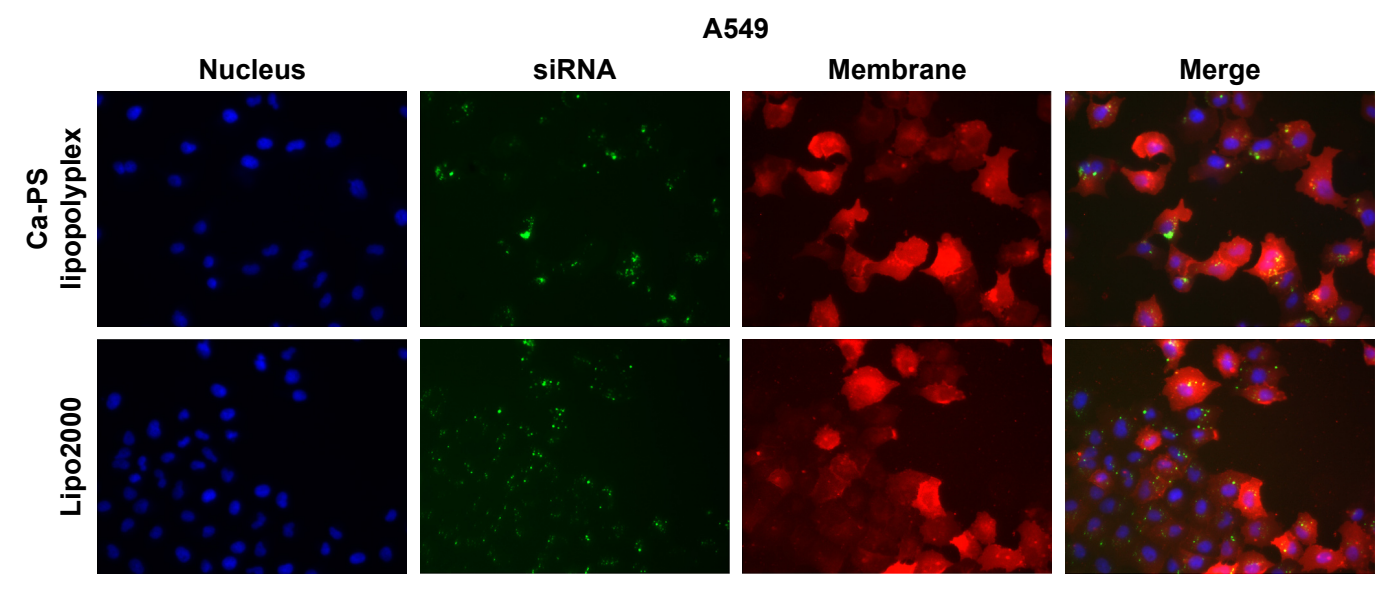

\section{HBE}
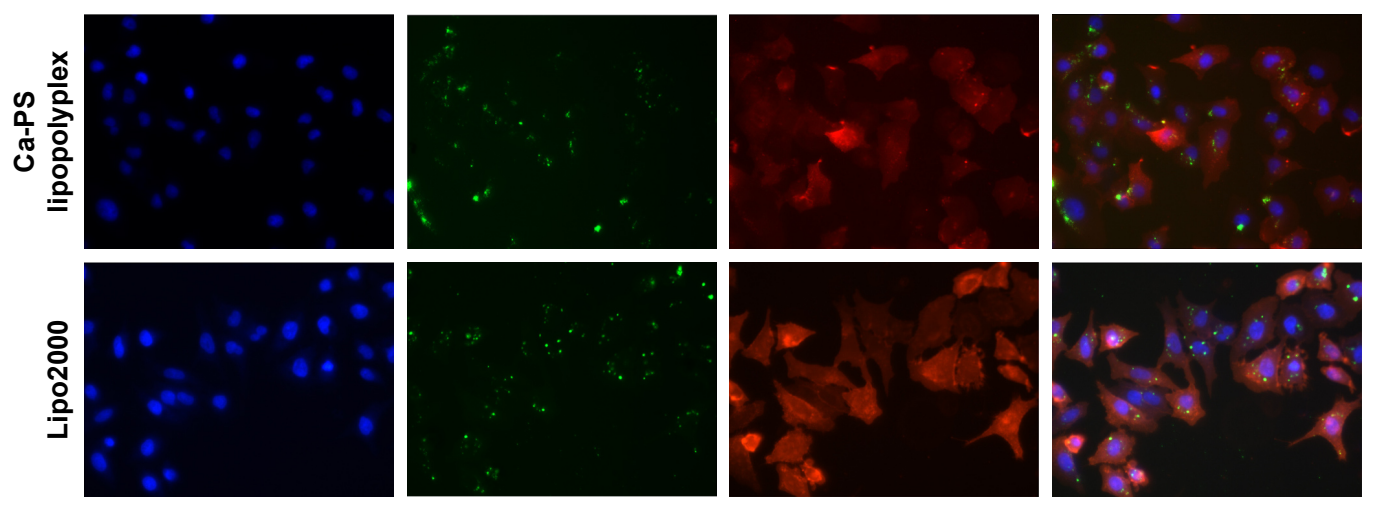

Ana-1
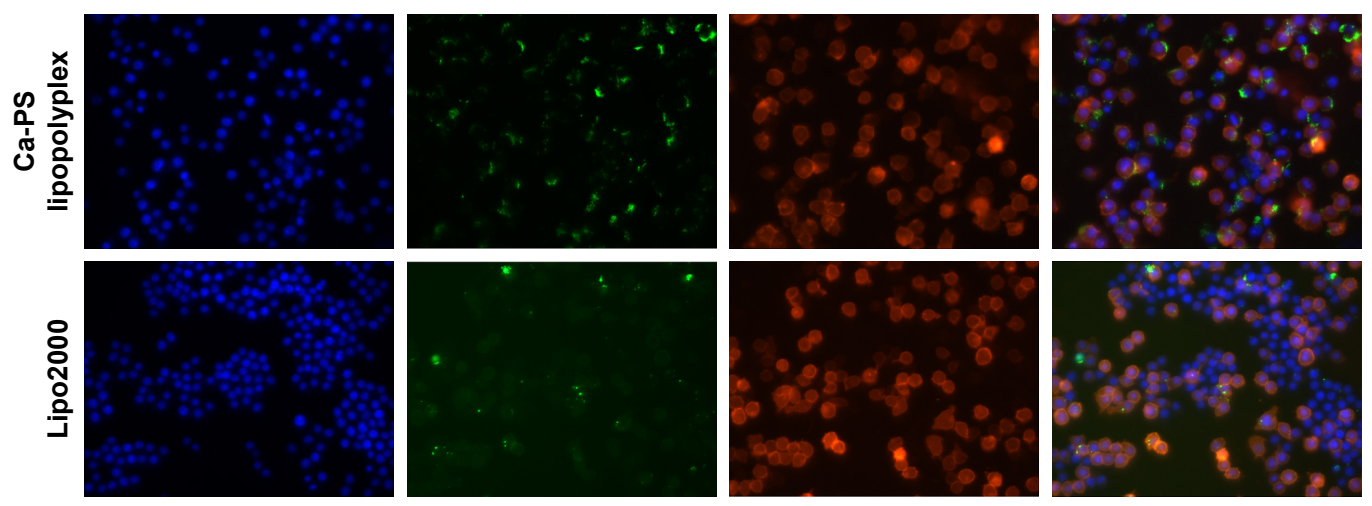

\section{BMDM}
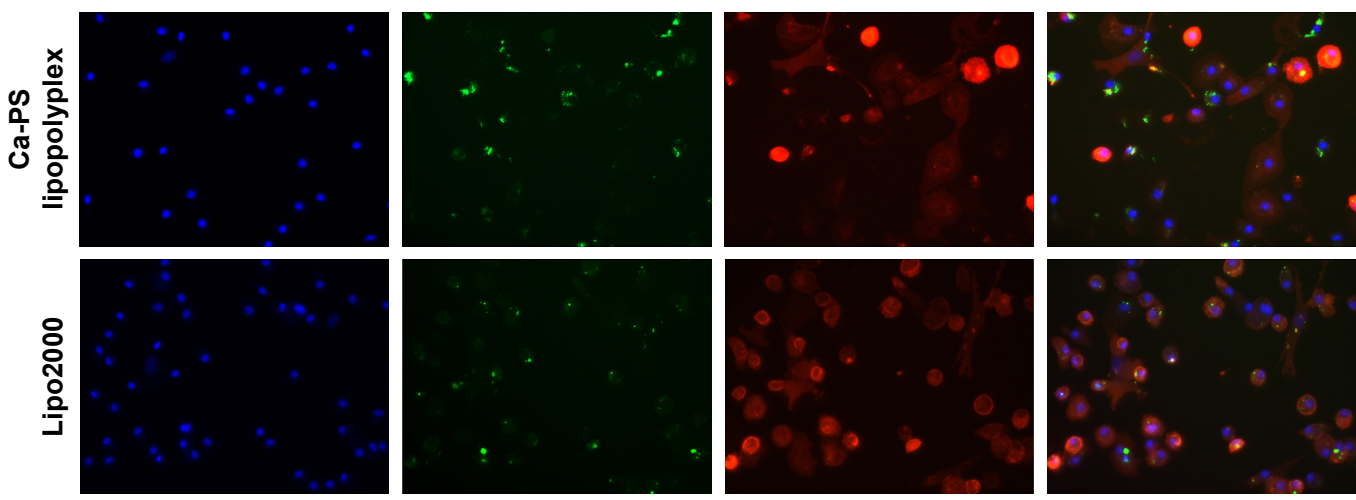

Figure 7 siRNA uptake of multiple cell types following transfection. A549, HBE, Ana-I, and BMDM were monitored for siRNA uptake upon transfection by Ca-PS lipopolyplex or Lipo2000 at 6 hours post-transfection.

Notes: FAM-siRNA was used as donor for green fluorescence. Nuclei and membrane of cells were stained by Hoechst 33,342 and Dil, respectively. Transfection type is indicated on the left of the graph, and cell type is indicated on the top of the images. 
In the last part of this section, we probed intracellular trafficking of siRNA in BMDMs upon transfection (Figure 8). Nuclei and lysosomes of BMDMs were stained by Hoechst 33,342 and Lyso-Tracker, respectively. BMDMs were imaged at 1, 6, 12, and 24 hours after transfection. As shown in Figure 8, uptake of siRNA by BMDM did not occur, regardless of the transfection type, at 1 hour post-transfection. However, at 6 hours post-transfection, Ca-PS lipopolyplex delivered significantly more siRNA than Lipo2000 to BMDM (enlarged images of Figure 8), which is consistent with Figure 7. Also, it is worth noting that siRNA co-localized with lysosomes in cytoplasm upon endocytosis into the cytoplasm at 6 hours post-transfection regardless of the transfection type, which indicates lysosomal/phagosomal entrapment of siRNA in BMDM. However, at 12 hours post-transfection, siRNAs delivered by Ca-PS lipopolyplex localized to the perinuclear regions (enlarged images). This observation suggests that siRNAs delivered by Ca-PS lipopolyplex escaped from lysosomes and clustered at the perinuclear regions where they exerted the post-transcriptional silencing effects. ${ }^{36,37}$ In contrast, siRNAs delivered by Lipo 2000 failed to move towards the nucleus and were entrapped in cytoplasmic locations far from the nucleus (enlarged images). siRNAs delivered by Lipo2000 were also characterized by markedly reduced fluorescence upon degradation within the lysosomes. These observations suggest that Ca-PS lipopolyplex promotes lysosomal escape and cytoplasmic siRNA trafficking towards the nucleus in BMDM. Lastly, at 24 hours post-transfection, siRNA fluorescence was barely observed in both transfection types, an outcome consistent with the results of Figure 6.

\section{Macrophage polarization}

In this part, we investigated whether macrophages are induced towards M1 or M2 polarization (activation) upon transfection by Ca-PS lipopolyplex carrying siNC. It is obvious that voluntary polarization of macrophage upon transfection is an obstacle towards macrophage research, especially when macrophage polarization is the research subject following genetic manipulation. Figure 9 shows the state of polarization of BMDM following siRNA transfection by Ca-PS lipopolyplex and Lipo2000 carrying siNCs. Employing antibodies to label surface markers of BMDM such as cd11b and F4/80, we detected a purity of $94.4 \%$ of extracted BMDM by flow cytometry as shown in Figure 9A. Figure 9B shows the state of polarization of BMDM 48 hours after transfection by Ca-PS lipopolyplex and Lipo2000. Majority of the population of the two positive controls (M1 and M2-polarized positive controls of BMDM induced by LPS and IL-4, respectively) is found within left two quadrants (M1 polarization) or the upper two quadrants (M2 polarization) compared to the untreated negative control. Treatments by both transfection methods, either Ca-PS lipopolyplex or Lipo2000, only resulted in a slight shift of population as compared to the untreated negative control. The percentage of M1 or M2-polarized BMDM cells of various treatment groups are shown in Figure 9C. It is apparent that siRNA-mediated transfection by Ca-PS lipopolyplex or Lipo2000 did not result in activation/polarization of BMDM towards either the M1 or M2 state. We also monitored polarization of Ana-1 upon transfection. Results are shown in Figure S2.

\section{Discussion}

The difficulty of transfecting immune cells has long been an obstacle for fast and reliable genetic manipulation in research pertinent to immunology. Immune cells are usually refractory to delivery of nucleotides by standard reagent-based methods such as lipid-based protocol. The use of viral vectors has been a worldwide consensus for transfecting immune cells. ${ }^{38,39}$ However, viral vector such as adenovirus and lentivirus are associated with biosafety issues that cannot be ignored. Human infection by these viruses, although engineered to be non-toxic, can still lead to harmful outcomes if viral genetic mutations give rise to re-acquirement of toxic attributes (eg, ability to replicate). Besides, preparation of viral vectors is time-consuming and error-prone. Genetic cloning, transfection of packaging cells (eg, 293T), collection of viral vectors, and determination of viral titer are all subject to mistakes. ${ }^{40}$ In contrast, liposomes are safe, cheap, and fast delivery systems for DNA and RNA delivery. Nevertheless, delivery of plasmids or siRNA to immune cells using liposomal formulas has yielded less than optimal results. In this work, we designed a liposomal formulation, namely Ca-PS lipopolyplex, to deliver siRNA to macrophages. The assembly of Ca-PS lipopolyplex is illustrated in Figure 10.

By simple incorporation of PS into the membrane, uptake of Ca-PS lipopolyplex by macrophages became much more favorable than standard cationic liposomal formulations lacking PS. Preliminary evaluation on the efficacy of Ca-PS lipopolyplex showed a higher knockdown efficiency of GFP in GFP-A549 cells compared to Lipo2000. However, knockdown of GAPDH in A549 and HBE cells using Ca-PS lipopolyplex didn't lead to statistically higher efficiency than Lipo2000. This may be explained by the housekeeping nature 


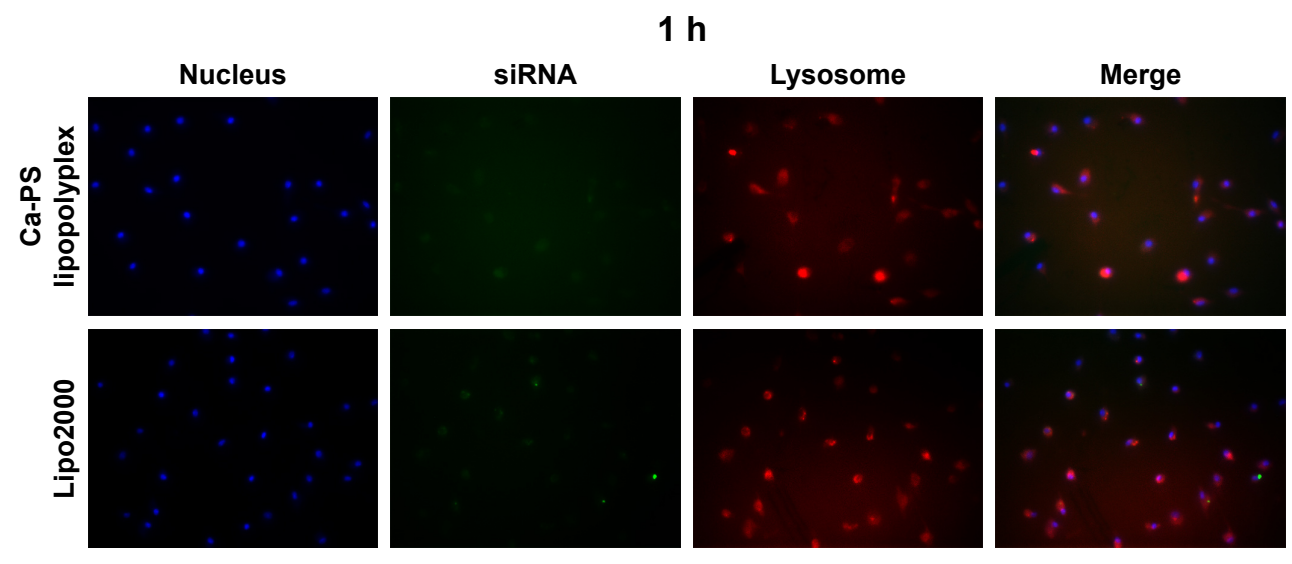

$6 \mathrm{~h}$
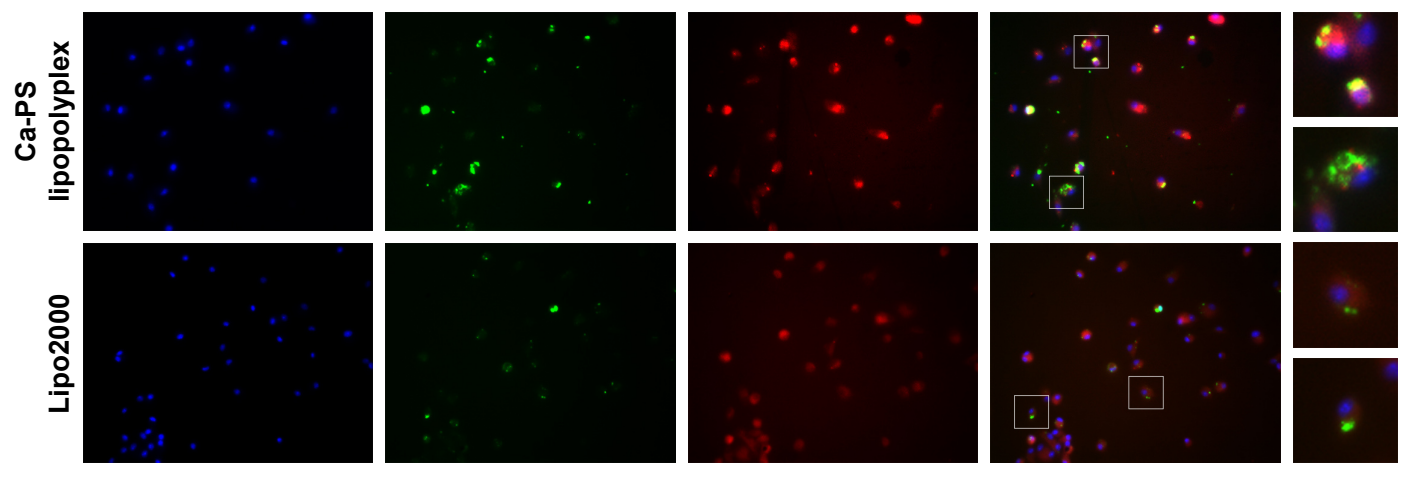

$12 \mathrm{~h}$
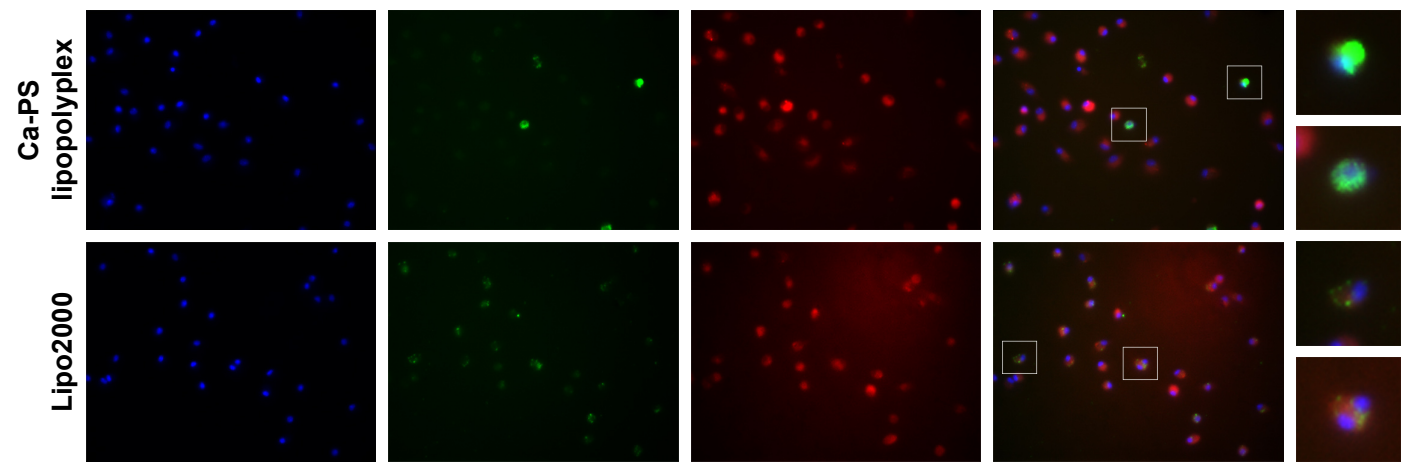

$24 \mathrm{~h}$
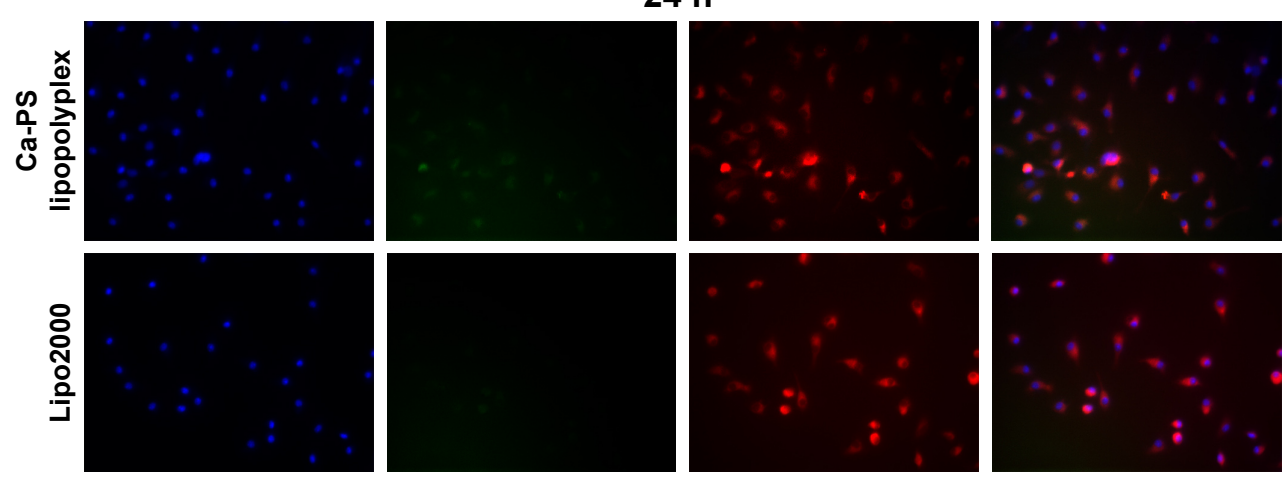

Figure 8 Intracellular trafficking of siRNA in BMDM. FAM-siRNA was used as donor for green fluorescence.

Notes: Nuclei and lysosomes of BMDM were stained by Hoechst 33,342 and LysoTracker, respectively. Transfection type is indicated on the left of the graph, and time post-transfection is indicated on the top of the images. For merged images at 6 hours and 12 hours post-transfection, white boxes within the pictures are enlarged to the right of each respective image to show details of siRNA localization. 

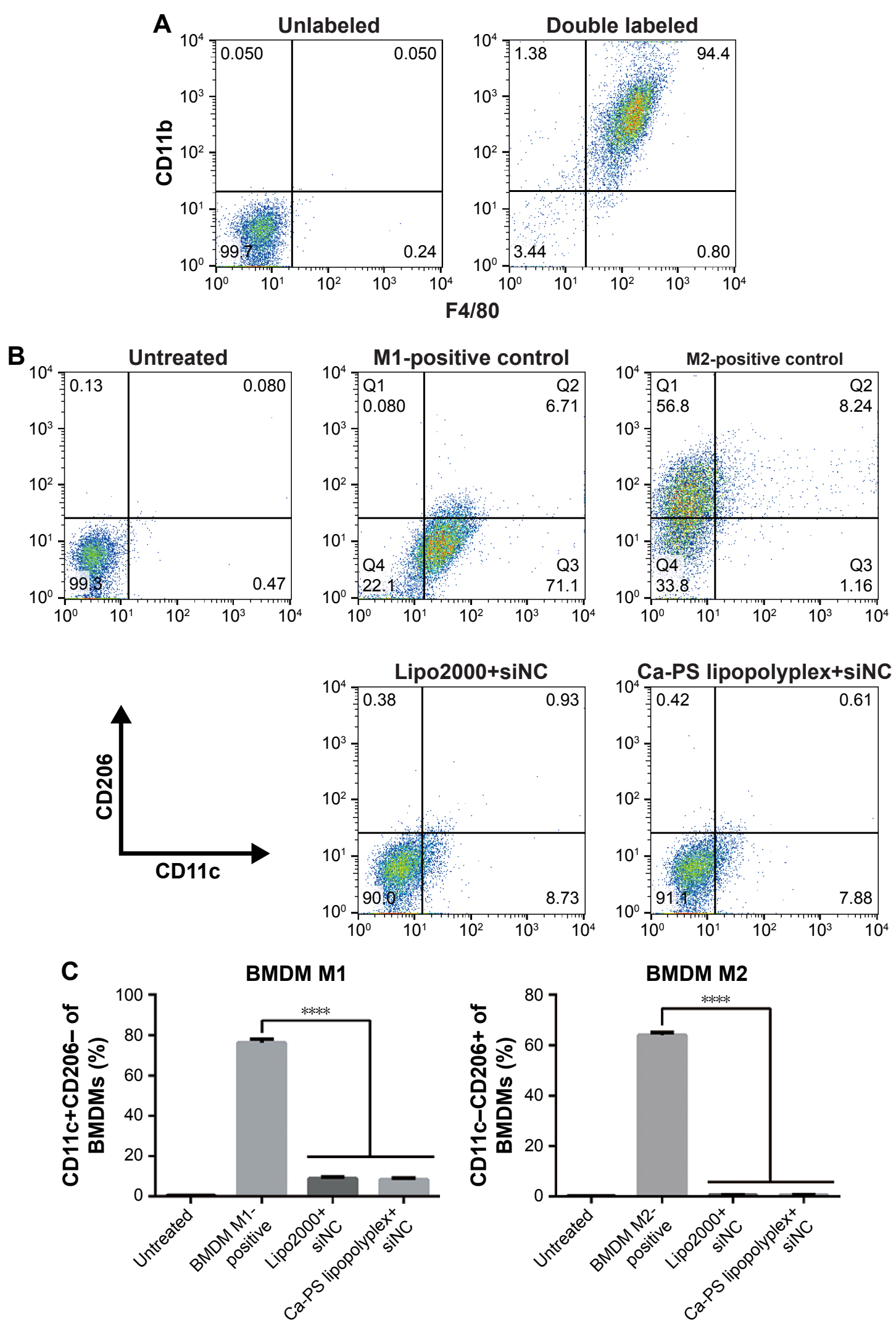

Figure 9 Polarization of BMDMs upon transfection by Ca-PS lipopolyplex.

Notes: (A) Purity of extracted BMDMs monitored by flow cytometry using F4/80 and CDIIb as surface markers. (B) Investigation on polarization of differentially treated BMDMs. CD206 and CDI Ic were used as surface markers to identify MI and M2-positive populations, respectively, of BMDMs upon transfection treatments. MI-polarized BMDMs were identified as CDI Ic+CD206- populations while M2-polarized BMDMs were identified as CDI Ic-CD206+ populations. MI and M2-positive controls were induced by LPS and IL-4, respectively, as positive controls. (C) Bar graphs showing percentage of MI or M2-polarized population of BMDMs upon various transfection treatments. $* * * * P<0.0001$. 


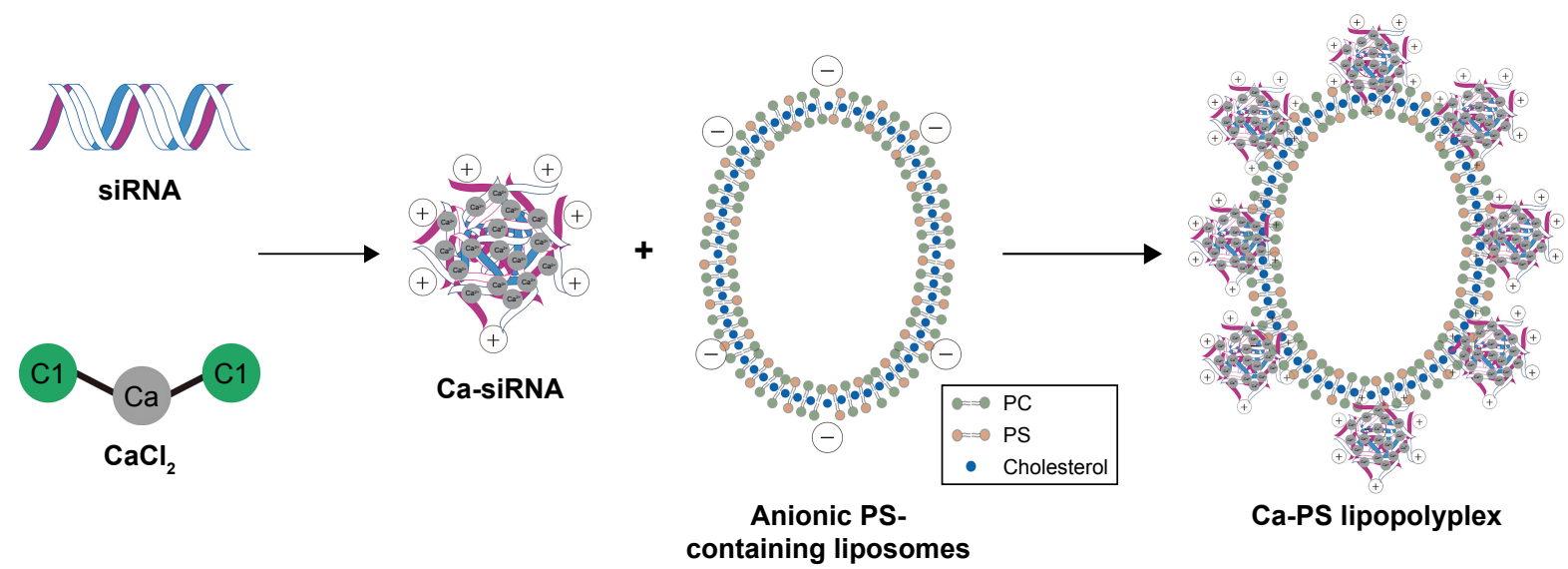

Figure 10 Illustration of the assembly of Ca-PS lipopolyplex.

Notes: Negatively charged siRNA first bind positively charged $\mathrm{Ca}^{2+}$ to form the $\mathrm{Ca}^{2+}$-siRNA complex, which in turn binds the anionic PS-containing liposomes to form the complete transfection-able Ca-PS lipopolyplex. PC, PS and chol units are indicated in the graph.

of GAPDH so that external knockdown of GAPDH triggered internal compensation to keep GAPDH at a relatively steady level. In the case of macrophages, both FADD and GAPDH genes were repressed at a substantially higher level by Ca-PS lipopolyplex than Lipo2000. This can be explained by the prominently higher uptake efficiency of the Ca-PS lipopolyplex by macrophages (Figures 6 and 7). A confirmation experiment was performed using EGFP-BMDMs. As expected, Ca-PS lipopolyplex achieved a higher knockdown efficiency of EGFP than Lipo2000. Statistically speaking, Ca-PS lipopolyplex was capable of accomplishing approximately a two-fold knockdown efficiency as compared to Lipo2000 in macrophages. We believe that Ca-PS lipopolyplex can be further optimized to achieve even higher efficiencies.

Uptake and intracellular trafficking of siRNAs packaged by Ca-PS lipopolyplex and Lipo2000 were also investigated in this study. Apart from profoundly improved uptake of siRNA, Ca-PS lipopolyplex promoted escape of siRNA from lysosomes/phagosomes of macrophages to allow implementation of gene silencing, both of which are key to efficient gene knockdown in macrophages.

The choice of using anionic formula for siRNA delivery is of profound significance in transfecting primary macrophages. As previously mentioned, cationic liposomes pose serious cytotoxicity to transfected cells. Unlike established cell lines, primary cells cannot be passaged indefinitely and therefore, are of very limited amounts. High cytotoxicity will reduce the amount of viable primary cells following transfection for further experiments. Consequently, anionic liposomes with low cytotoxicity, namely PS-bearing liposomes in our study, are advantageous in delivering siRNA to primary cells.
The biology of immune cells endows them with the mechanics to destroy deleterious and foreign substances and therefore, DNAs or RNAs carried by any delivery system are prone to destruction before taking effect in target cells. Our success with Ca-PS lipopolyplex in macrophages can indeed be extended to other immune cells such as T cells. Although $\mathrm{T}$ cells may not be exploited in a way similar to professional phagocytes such as macrophages, the immunosuppressive role of PS can contribute to protection of materials packed by liposomes to avoid premature destruction by T cells. ${ }^{41}$

\section{Conclusion}

In this study, we used a liposomal mimic of apoptotic bodies to deliver siRNAs to the hard-to-transfect macrophages. DOPS contained within our anionic liposomes mimiced the membrane of apoptotic bodies and was expected to significantly improve uptake of the transfection complex by macrophages. The Ca-PS lipopolyplex showed transfection competence of siRNAs in multiple cell types. Moreover, Ca-PS lipopolyplex showed decreased cytotoxicity compared to Lipo2000 in A549 cells, possibly due to its anionic nature. In macrophages, namely Ana-1 and BMDMs in this study, siRNAs targeting FADD, GAPDH, and EGFP delivered by Ca-PS lipopolyplex exhibited substantially improved knockdown efficiency compared to Lipo2000, recording an average improvement higher than $100 \%$. Further studies on siRNA uptake, intracellular trafficking, and macrophage polarization provided biological foundations and evidence of suitability for application of Ca-PS lipopolyplex in macrophages.

In summary, Ca-PS lipopolyplex is an anionic, stable, and non-toxic liposomal transfection reagent suitable for siRNA application in multiple cell types. Most importantly, 
Ca-PS lipopolyplex, which is a mimic of apoptotic bodies, has a profound advantage over standard cationic liposomal transfection reagent in effecting siRNA-mediated knockdown of genes in macrophages.

\section{Abbreviations}

ApoBDs, apoptotic bodies; BMDMs, bone-marrow derived macrophages; Chol, cholesterol; DLS, dynamic light scattering; DOPC, 1, 2-dioleoyl-sn-glycero-3-phosphocholine; DOPS, 1, 2-dioleoyl-sn-glycero-3-phosphatidylserine; FAM, fluorescent dye; IL-4, interleukin 4; LPS, lipopolysaccharides; MCSF, macrophage colony-stimulating factor; MST, microscale thermophoresis; NSCLC, non-small-cell lung cancer; PDI, polydispersity index; PC, phosphocholine; PS, phosphatidylserine; qPCR, quantitative realtime polymerase chain reaction; siNC, siRNA negative control.

\section{Acknowledgments}

The authors would like express their appreciation to Professor Can Zhang at China Pharmaceutical University for her invaluable inputs to the Discussion section. This study was supported in part by grants from the National Natural Science Foundation of China (81630092, 81773099, 81570790, 81573338), the National Key R\&D Research Program by Ministry of Science and Technology (2017YFA0506002, 2017YFA0104301, 2016YFC0902700) and Shenzhen Science and Technology Innovation Committee (JCYJ20160331152141936), Shenzhen Peacock Plan (KQTD20140630165057031).

\section{Ethics statement}

Animal care and use were performed strictly in accordance with the ethical guidelines of the Nanjing University Animal Care and Use Committee, and the experimental protocol was approved by the review board of the Nanjing University Animal Care and Use Committee.

\section{Author contributions}

All authors contributed to data analysis, drafting and revising the article, gave final approval of the version to be published, and agree to be accountable for all aspects of the work.

\section{Disclosure}

Zichun Hua is the founder and CEO of Jiangsu Target Pharma Laboratories Inc. The authors report no other conflicts of interest in this work.

\section{References}

1. Fire A, Xu S, Montgomery MK, Kostas SA, Driver SE, Mello CC. Potent and specific genetic interference by double-stranded RNA in Caenorhabditis elegans. Nature. 1998;391(6669):806-811.

2. Hughes J, Yadava P, Mesaros R. Liposomal siRNA delivery. Methods Mol Biol. 2010;605:445-459.

3. Landen CN, Merritt WM, Mangala LS, et al. Intraperitoneal delivery of liposomal siRNA for therapy of advanced ovarian cancer. Cancer Biol Ther. 2006;5(12):1708-1713.

4. Lin Q, Chen J, Zhang Z, Zheng G. Lipid-based nanoparticles in the systemic delivery of siRNA. Nanomedicine. 2014;9(1):105-120.

5. Mccaskill J, Singhania R, Burgess M, et al. Efficient biodistribution and gene silencing in the lung epithelium via intravenous liposomal delivery of siRNA. Mol Ther Nucleic Acids. 2013;2:e96.

6. Sakurai Y, Hatakeyama H, Sato Y, Hyodo M, Akita H, Harashima H. Gene silencing via RNAi and siRNA quantification in tumor tissue using MEND, a liposomal siRNA delivery system. Mol Ther. 2013;21(6): 1195-1203.

7. Sato Y, Hatakeyama H, Sakurai Y, Hyodo M, Akita H, Harashima H. A pH-sensitive cationic lipid facilitates the delivery of liposomal siRNA and gene silencing activity in vitro and in vivo. J Control Release. 2012; 163(3):267-276.

8. Schroeder A, Levins CG, Cortez C, Langer R, Anderson DG. Lipidbased nanotherapeutics for siRNA delivery. J Intern Med. 2010;267(1): 9-21.

9. Whitehead KA, Langer R, Anderson DG. Knocking down barriers: advances in siRNA delivery. Nat Rev Drug Discov. 2009;8(2):129-138.

10. Zhang S, Zhi D, Huang L. Lipid-based vectors for siRNA delivery. J Drug Target. 2012;20(9):724-735.

11. Dokka S, Toledo D, Shi X, Castranova V, Rojanasakul Y. Oxygen radical-mediated pulmonary toxicity induced by some cationic liposomes. Pharm Res. 2000;17(5):521-525.

12. Filion MC, Phillips NC. Toxicity and immunomodulatory activity of liposomal vectors formulated with cationic lipids toward immune effector cells. Biochim Biophys Acta. 1997;1329(2):345-356.

13. Kermanizadeh A, Jacobsen NR, Roursgaard M, Loft S, Møller P. Hepatic toxicity assessment of cationic liposome exposure in healthy and chronic alcohol fed mice. Heliyon. 2017;3(11):e00458.

14. Lappalainen K, Jääskeläinen I, Syrjänen K, Urtti A, Syrjänen S. Comparison of cell proliferation and toxicity assays using two cationic liposomes. Pharm Res. 1994;11(8):1127-1131.

15. Masotti A, Mossa G, Cametti C, et al. Comparison of different commercially available cationic liposome-DNA lipoplexes: Parameters influencing toxicity and transfection efficiency. Colloids Surf B Biointerfaces. 2009;68(2):136-144.

16. Nagahiro I, Mora BN, Boasquevisque CH, Scheule RK, Patterson GA. Toxicity of cationic liposome-DNA complex in lung isografts. Transplantation. 2000;69(9):1802-1805.

17. Schreier H, Gagné L, Bock T, et al. Physicochemical properties and in vitro toxicity of cationic liposome cDNA complexes. Pharm Acta Helv. 1997;72(4):215-223.

18. Zhong YQ, Wei J, Fu YR, et al. Toxicity of cationic liposome Lipofectamine 2000 in human pancreatic cancer Capan-2 cells. Nan Fang Yi Ke Da Хие Хие Bao. 2008;28(11):1981-1984.

19. Collins DE, Reuter JD, Rush HG, Villano JS. Viral vector biosafety in laboratory animal research. Comp Med. 2017;67(3):215-221.

20. Athlin L, Holmberg SB, Hafström L. Macrophage function and surgery. A clinical review with special reference to phagocytosis. Eur J Surg. 1991;157(3):163-170.

21. Binns WH. The macrophage - phagocytosis and pinocytosis: a review. Bull Phila Cty Dent Soc. 1967;32(8):10-13.

22. Chockalingam S, Ghosh SS. Macrophage colony-stimulating factor and cancer: a review. Tumour Biol. 2014;35(11):10635-10644.

23. Horwood NJ. Macrophage polarization and bone formation: a review. Clin Rev Allergy Immunol. 2016;51:79-86. 
24. Peter C, Waibel M, Radu CG, et al. Migration to apoptotic "find-me" signals is mediated via the phagocyte receptor G2A. J Biol Chem. 2008; 283(9):5296-5305.

25. Gardai SJ, Bratton DL, Ogden CA, Henson PM. Recognition ligands on apoptotic cells: a perspective. J Leukoc Biol. 2006;79(5):896-903.

26. Geelen T, Yeo SY, Paulis LE, Starmans LW, Nicolay K, Strijkers GJ. Internalization of paramagnetic phosphatidylserine-containing liposomes by macrophages. J Nanobiotechnology. 2012;10:37.

27. Zhang X, Goncalves R, Mosser DM. The isolation and characterization of murine macrophages. Curr Protoc Immunol. 2008; Chapter 14: Unit14.1.

28. Wienken CJ, Baaske P, Rothbauer U, Braun D, Duhr S. Protein-binding assays in biological liquids using microscale thermophoresis. Nat Commun. 2010;1:100.

29. Patil SD, Rhodes DG, Burgess DJ. Anionic liposomal delivery system for DNA transfection. AAPS J. 2004;6(4):e29.

30. Ruvinov E, Kryukov O, Forti E, Korin E, Goldstein M, Cohen S. Calcium-siRNA nanocomplexes: what reversibility is all about. J Control Release. 2015;203:150-160.

31. Jud W, Virden JCB. Sodium chloride-induced aggregation of dipalmitoylphoshpatidylglycerol small unilamellar vesicles with varying amounts of incorporated cholesterol. Langmuir. 1992;8:1532-1537.

32. Lee C, Barnett J, Reaven PD. Liposomes enriched in oleic acid are less susceptible to oxidation and have less proinflammatory activity when exposed to oxidizing conditions. J Lipid Res. 1998;39(6):1239-1247.

33. Papahadjopoulos D, Jacobson K, Nir S, Isac T. Phase transitions in phospholipid vesicles. Fluorescence polarization and permeability measurements concerning the effect of temperature and cholesterol. Biochim Biophys Acta. 1973;311(3):330-348.
34. Gibbons A, McElvaney NG, Cryan SA. A dry powder formulation of liposome-encapsulated recombinant secretory leukocyte protease inhibitor (rSLPI) for inhalation: preparation and characterisation. AAPS PharmSciTech. 2010;11(3):1411-1421.

35. Kelly C, Jefferies C, Cryan SA. Targeted liposomal drug delivery to monocytes and macrophages. J Drug Deliv. 2011:727241.

36. Berezhna SY, Supekova L, Supek F, Schultz PG, Deniz AA. siRNA in human cells selectively localizes to target RNA sites. Proc Natl Acad Sci US A. 2006;103(20):7682-7687.

37. Rao DD, Vorhies JS, Senzer N, Nemunaitis J. siRNA vs. shRNA: similarities and differences. Adv Drug Deliv Rev. 2009;61(9):746-759.

38. Bonini C, Ferrari G, Verzeletti S, et al. HSV-TK gene transfer into donor lymphocytes for control of allogeneic graft-versus-leukemia. Science. 1997;276(5319):1719-1724.

39. Pule MA, Savoldo B, Myers GD, et al. Virus-specific T cells engineered to coexpress tumor-specific receptors: persistence and antitumor activity in individuals with neuroblastoma. Nat Med. 2008;14(11): 1264-1270.

40. Srinivasakumar N. Packaging cell system for lentivirus vectors. Preparation and use. Methods Mol Med. 2002;69:275-302.

41. Birge RB, Boeltz S, Kumar S, et al. Phosphatidylserine is a global immunosuppressive signal in efferocytosis, infectious disease, and cancer. Cell Death Differ. 2016;23(6):962-978. 


\section{Supplementary materials}

Table SI siRNA sequences

\begin{tabular}{l|l}
\hline mGapdh & $5^{\prime}$-CACUCAAGAUUGUCAGCAATT-3' \\
hGapdh & $5^{\prime}$-GUAUGACAACAGCCUCAAGTT-3' \\
mFADD & $5^{\prime}$-ACGAUCUGAUGGAGCUCAA-3' \\
hFADD & $5^{\prime}$-GAAGACCUGUGUGCAGCAUTT-3' \\
siEGFP and siGFP & $5^{\prime}$-GGCACAAGCUGGAGUACAACU-3' \\
siNC and FAM-siNC & $5^{\prime}$-UUCUCCGAACGUGUCACGUTT-3' \\
\hline
\end{tabular}

Table S2 Primer sequences

\begin{tabular}{l|l}
\hline Mouse Actin $F$ & GAGACCTTCAACACCCCAGC \\
Mouse Actin R & ATGTCACGCACGATTTCCC \\
Human Actin F & GTGACAGCAGTCGGTTGGAG \\
Human Actin R & AGTGGGGTGGCTTTTAGGAT \\
Mouse GAPDH F & TCTCCCTCACAATTTCCATCCCAG \\
Mouse GAPDH R & GGGTGCAGCGAACTTTATTGATGG \\
Human GAPDH F & ATGTACGTAGCCATCCAGGC \\
Human GAPDH R & AGGAAGGAAGGCTGGAAGAG \\
Human FADD F & CACAGACCACCTGCTTCTGA \\
Human FADD R & CTGGACACGGTTCCAACTTT \\
Mouse FADD F & CGGGCAACGATCTGATGGA \\
Mouse FADD R & ACAATGTCAAATGCCACCTGC \\
\hline
\end{tabular}
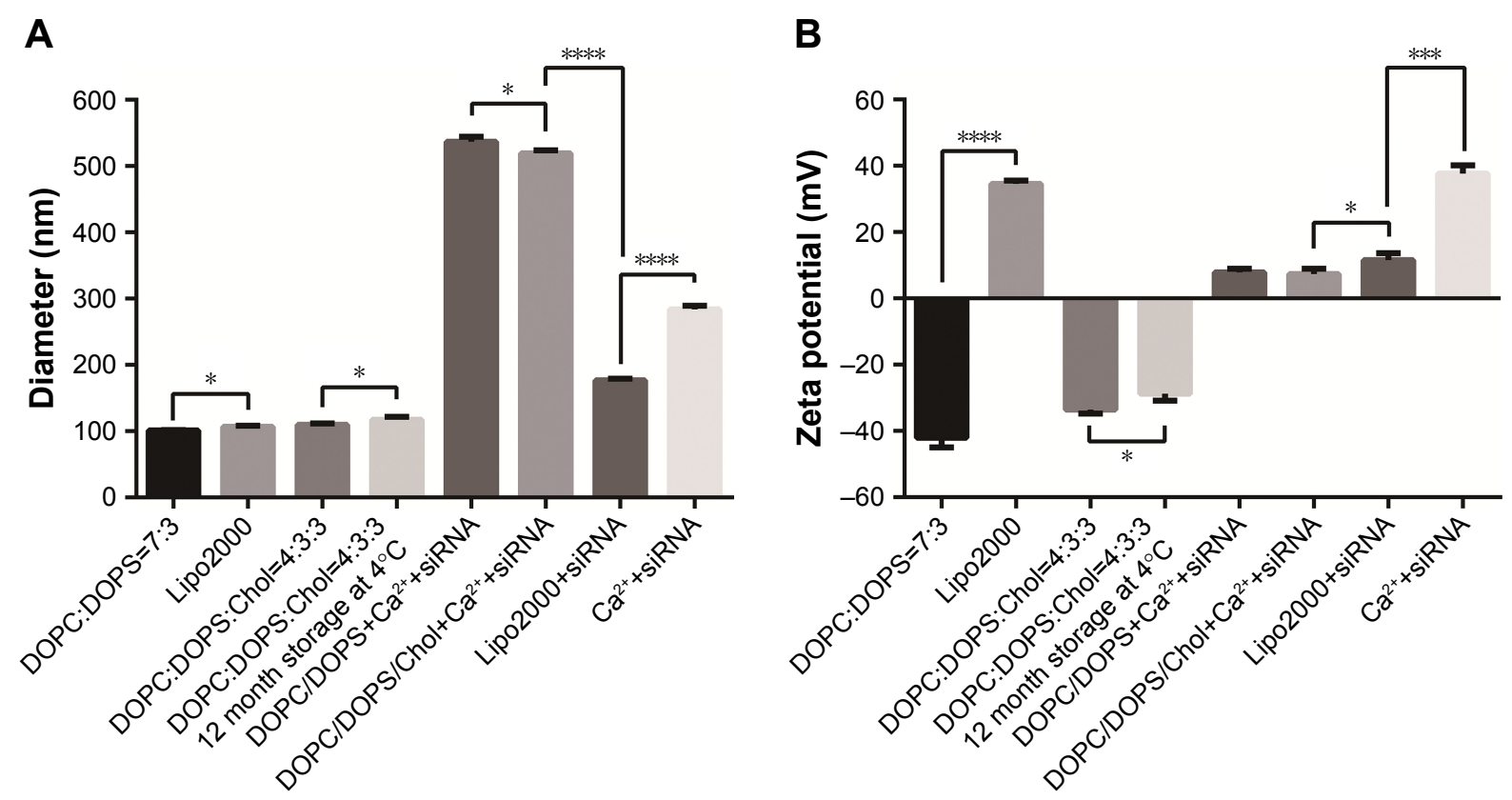

Figure SI Bar graphs representative of data in Table 2 showing size and Zeta potential of various particles. Notes: (A) Particle diameters. (B) Zeta potentials. $* P<0.05$, $* * * P<0.00$ I, and $* * * * P<0.0001$. 

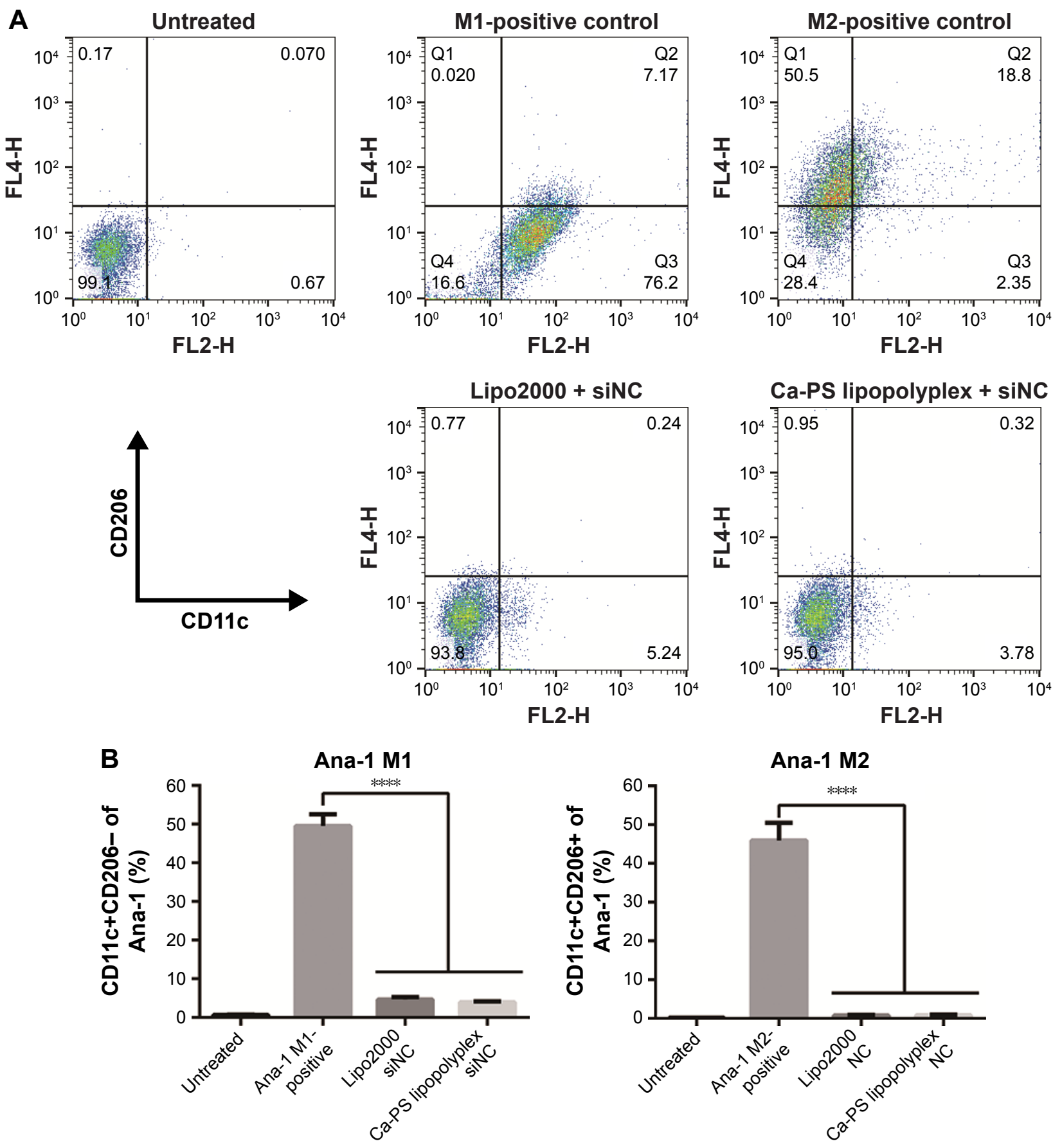

Figure S2 Polarization of Ana-I upon transfection by Ca-PS lipopolyplex.

Notes: (A) Investigation on polarization of differentially treated Ana-I. CD206 and CDIIc were used as surface markers to identify MI and M2-positive populations, respectively, of Ana-I upon transfection treatments. MI-polarized Ana-I cells were identified as CDI Ic+CD206- populations while M2-polarized Ana-I cells were identified as CDI Ic-CD206+ populations. MI and M2-positive controls were induced by LPS and IL-4, respectively, as positive controls. (B) Bar graphs showing percentage of MI or M2-polarized population of Ana-I upon various transfection treatments. $* * * * P<0.000 I$.

International Journal of Nanomedicine

\section{Publish your work in this journal}

The International Journal of Nanomedicine is an international, peerreviewed journal focusing on the application of nanotechnology in diagnostics, therapeutics, and drug delivery systems throughout the biomedical field. This journal is indexed on PubMed Central, MedLine, CAS, SciSearch $®$, Current Contents ${ }^{\circledR} /$ Clinical Medicine,

Journal Citation Reports/Science Edition, EMBase, Scopus and the Elsevier Bibliographic databases. The manuscript management system is completely online and includes a very quick and fair peer-review system, which is all easy to use. Visit http://www.dovepress.com/ testimonials.php to read real quotes from published authors. 